\title{
Quand les morts reviennent... Réflexion sur l'ancestralité chez les Mayas des Basses Terres
}

Olivier Le Guen

\section{(2) OpenEdition}

\section{Journals}

Édition électronique

URL : https://journals.openedition.org/jsa/1560

DOI : 10.4000/jsa.1560

ISSN : 1957-7842

\section{Éditeur}

Société des américanistes

\section{Édition imprimée}

Date de publication : 5 juin 2003

Pagination : 171-205

ISSN : 0037-9174

\section{Référence électronique}

Olivier Le Guen, « Quand les morts reviennent... Réflexion sur l'ancestralité chez les Mayas des

Basses Terres », Journal de la Société des américanistes [En ligne], 89-2 | 2003, mis en ligne le 05 juin 2008, consulté le 04 septembre 2022. URL : http://journals.openedition.org/jsa/1560 ; DOI : https:// doi.org/10.4000/jsa. 1560 


\title{
QUAND LES MORTS REVIENNENT... RÉFLEXION SUR L'ANCESTRALITÉ CHEZ LES MAYAS DES BASSES TERRES
}

\author{
Olivier LE GUEN *
}

\begin{abstract}
Le culte des ancêtres est souvent évoqué dans la littérature ethnologique amérindienne, sans que soit pourtant établie son existence. Cet article s'attache à montrer que certains groupes mayas des Basses Terres pratiquent un culte familial des ancêtres, dont le jour des morts, ou hanal pixan, apparaît comme la manifestation la plus prégnante. Nous fonderons notre raisonnement sur des critères précis empruntés à des études ethnologiques de sociétés dites "à ancêtres » auxquels seront confrontées les croyances et les pratiques mayas. L'approche est comparative, à la fois dans le temps, en croisant des données coloniales et ethnographiques du $\mathrm{xx}^{\mathrm{e}}$ siècle, et dans l'espace, en confrontant les usages et les croyances de deux groupes mayas pourtant apparentés, les Yucatèques et les Lacandons. [MoTS CLÉs : Mayas-Yucatèques, Lacandons, morts, ancêtres, hanal pixan, culte des ancêtres.]
\end{abstract}

When the dead come home. Remarks on ancestor worship among the Lowland Mayas. In Amerindian ethnographical literature, ancestor worship is often mentioned but evidence of its existence is lacking. This article will try to demonstrate that some Lowland Maya do worship ancestors ; it will use precise criteria taken from ethnological studies of societies where ancestor worship is common, compared to maya beliefs and practices. The All Souls' Day, or hanal pixan, seems to be the most significant manifestation of this cult. Our approach will be comparative, through time - using colonial and ethnographical data of the twentieth century, and space - contemplating uses and beliefs of two maya groups, the Yucatec and the Lacandon Maya. [KeY wORDS : Yucatec Maya, Lacandon, dead, ancestors, hanal pixan, ancestor worship.]

Cuando los muertos vuelven... Reflexión sobre la ancestralidad entre los mayas de las tierras bajas. En la literatura etnológica amerindia, se evoca muchas veces el culto de los antepasados, pero nunca fue asentada su existencia. En este artículo, queremos demostrar que ciertos grupos mayas de las tierras bajas practican un culto a los antepasados, en el cual el día de los muertos, o hanal pixan, aparece como una manifestación significativa. La reflexión sobre partirá de precisos sacados de estudios etnológicos de sociedades donde se practica el culto de los antepasados, de los cuales se cotejarán con las creencias y las prácticas de los mayas. Nuestro enfoque es comparativo tanto en el tiempo, ya que utiliza datos de crónicas coloniales y de la etnografía del siglo veinte,

* Doctorant en ethnologie, Laboratoire d'ethnologie et de sociologie comparative, UMR 7535, Maison de l'archéologie et de l'ethnologie, Université Paris X-Nanterre [olivierleguen@free.fr].

Journal de la Société des Américanistes, 2003, 89-2, pp. 171-205. CSociété des Américanistes. 
como en el espacio, puesto que confronta los usos y las creencias de dos grupos mayas, los yucatecos y los lacandones. [PALABRAS CLAves : mayas-yucatecos, lacandones, muertos, ancestros, hanal pixan, culto de los antepasados.]

\section{INTRODUCTION}

Dans tous les villages mayas de la péninsule du Yucatán, le murmure des prières pour les morts résonne le soir du 31 octobre. Depuis quelques jours, les festivités se préparent en vue de cette semaine tant attendue pendant laquelle les morts yucatèques sont conviés à manger parmi les vivants. Cet événement annuel, connu sous le nom de hanal pixan - expression signifiant « la nourriture des âmes »-, est célébré avec une ferveur qu'on peut hésiter à qualifier de " catholique ». En effet, bien que la date du hanal pixan corresponde à un jour près à celle de la commémoration des morts dans le calendrier liturgique catholique, cette coutume renferme, sous ses apparences chrétiennes, une origine bien antérieure à la conquête espagnole. Nous pensons que ce rite du $1^{\text {er }}$ novembre peut être interprété, non pas simplement comme un hommage rendu aux morts, mais bien comme un culte des ancêtres. Toutefois, si cette célébration est fondamentale pour les habitants de la péninsule du Yucatán, elle est totalement absente chez les Mayas-Lacandons du Chiapas, pourtant très proches linguistiquement, historiquement et culturellement de ceux du Yucatán.

Le débat sur la différence entre les morts et les ancêtres est déjà ancien. Au début du $\mathrm{xx}^{\mathrm{e}}$ siècle, Tylor (1920) considère le culte des ancêtres comme la forme archétypale de la religion primitive. Pour Durkheim, les premiers rites sont des rites funéraires et les premiers autels, des tombes. Les principales questions qui animent alors les ethnologues sont celles de la genèse de ce rituel et du caractère bénéfique ou maléfique de la figure de l'ancêtre. Les premières définitions proposées ne sont guère satisfaisantes, ainsi celle de Durkheim qui dit, dans Les Formes élémentaires de la vie religieuse (1912), qu'il n'y a pas de culte des ancêtres sauf lorsque des sacrifices sont faits sur les tombes de temps en temps, quand des libations y sont versées à certaines dates plus ou moins spécifiques, ou quand des fêtes sont régulièrement célébrées en l'honneur des morts. Durkheim ne réussit pas à distinguer le culte des morts de celui des ancêtres. Pour Hertz (1970), si le premier existe dans toutes les sociétés, le second, en revanche, est moins répandu. Il faut attendre les années 1960 et 1970 pour que les ethnologues tranchent vraiment la question en tentant d'apporter des définitions à partir d'études ethnographiques menées en Afrique (Goody 1962 ; Fortes 1965) et dans divers pays d'Asie comme la Chine (Freedman 1970; Ahern 1973 ; Wolf 1974), Taiwan (Jordan 1972) ou le Japon (Smith 1974). Toutefois, il n'existe pas, à notre connaissance, de telles études en Méso-Amérique et, en particulier, chez les Mayas des Basses Terres. Dans l'ethnographie des divers groupes mayas, il est 
parfois fait mention d'un culte des ancêtres qui se caractérise par son aspect collectif. C'est le cas, par exemple, des morts des Tzotzil, les totilme'iletik (pèresmères) que Vogt $(1966$, p. 282) qualifie de "déités ancestrales et tribales » (" ancestral ", " tribal » deities), insistant sur leur caractère communautaire. Le culte rendu en leur honneur est donc particulier, réalisé par des groupes cérémoniels, composés de quelques personnes du village. Il en va de même pour les K'iche' du Guatemala chez qui, lors de cérémonies, on invoque collectivement tous les ancêtres du groupe (Breton 1991).

Notre travail partira de l'étude d'un culte « familial » où l'ancêtre est avant tout considéré comme un ascendant familial direct. Il ne s'agira pas d'opposer deux formes de culte, mais plutôt de discerner deux types de rituels réunissant des groupes sociaux distincts - la communauté tout entière dans un cas; la famille et les amis proches dans l'autre - et proposant une catégorisation différente de l'ancêtre - ancêtres de la communauté ou héros culturels d'une part ; ascendants familiaux d'autre part. Dans la littérature mayaniste, les termes « morts » et « ancêtres » sont parfois employés indistinctement et ne sont à aucun moment définis. C'est pourquoi il est nécessaire de préciser les caractéristiques générales de la figure de l'ancêtre ainsi que celles du culte qui lui est destiné. Pour cela, nous ferons appel aux études effectuées dans des sociétés dites « à ancêtres »en Afrique ou en Asie.

En premier lieu, remarquons que les morts ne sont pas tous des ancêtres. Des rites spécifiques, en plus de l'inhumation, sont souvent nécessaires pour conférer le statut d'ancêtre (Hertz 1970) et faire du mort une entité identifiable et individualisée. Par exemple, pour les Tallensi, cette individualisation se fait par le nom ; Fortes (1965, p. 124) parle d'une véritable « généonomie ». En Chine, lorsque les ancêtres provoquent des troubles parmi les vivants, ils sont identifiés par la divination et restent de cette manière dans le souvenir des hommes (Wolf 1974). Dans la plupart des sociétés pratiquant un tel culte, une relique est parfois présente, elle sert à la spécification du mort et permet de le vénérer. En Asie (Chine, Japon et Taiwan), c'est une tablette de bois gravée, placée dans un hall, qui permet d'entrer en contact avec l'âme du défunt. Dans les Andes, la momie d'un ancêtre fait l'objet d'un culte, celui de l'ayllu, que l'on connaît grâce à des traces archéologiques et quelques récits coloniaux (Salomon 1991 cité dans McAnany 1995, p. 17). Pour Krickeberg (1962, p. 75),

[on] ne peut pas parler chez les Aztèques d'un culte des ancêtres tel que celui qui existait au Pérou, bien que, lors de la fète des morts, ils dressaient pour leurs guerriers tombés à l'ennemi ou sacrifiés par lui une momie revêtue des attributs de Mixcoatl et, pour le défunt roi, une statue de bois habillée à la façon de Quetzalcoatl, puis sacrifiassent (sic) devant elles.

De nombreux auteurs (Goody 1962 ; Fortes 1965 ; Freedman 1966) insistent sur le fait que l'ancêtre, contrairement au mort, intervient dans les affaires quotidiennes des vivants. 
En second lieu, soulignons le fait que le culte des morts et celui des ancêtres présentent des différences notables. Si le premier, tel qu'il existe en Europe, n'est qu'un simple rite de commémoration, le second, en revanche, est un acte de reconnaissance-vénération des aïeuls par le biais d'offrandes (nourriture, sang), de libations, d'objets matériels, de prières et de quelque marque de respect. Ces offrandes ne sont pas de simples cadeaux mais des obligations auxquelles les vivants sont contraints de se soumettre, afin d'éviter la colère de ces morts particuliers (Goody 1962 ; Wolf 1974). Le lien de filiation est au cœur du culte des ancêtres puisque ceux-ci sont définis par rapport à une lignée (Fortes 1965). Il arrive toutefois que des ancêtres sans descendance soient vénérés : c'est le cas au Japon (Smith 1974). Quoi qu'il en soit, ce culte est enraciné dans les institutions familiales et dans la parenté, c'est d'ailleurs un devoir qui revient souvent aux descendants. Le culte des ancêtres sert parfois - comme chez les Tallensi (Fortes 1965) - à renforcer l'autorité juridique et le droit légitime ; il permet un contrôle sur les relations sociales du groupe. Dans d'autres cas, il est lié à l'héritage. En Afrique comme en Chine, il s'agit des terres (Goody 1962; Ahern 1973), alors qu'au Japon il peut s'agir de droits (Smith 1974). Afin d'éclairer davantage le propos, on rappellera cette définition de Fortes qui, malgré sa concision, a le mérite de dégager les principaux critères qui caractérisent le culte des ancêtres par opposition au culte des morts: "Un "esprit" ancestral n'est pas pensé comme un être nébuleux ou une présence mystique personnifiée mais d'abord comme un nom attaché à une relique [...] représentant une validation rituelle de l'ancestralité du lignage et une intervention mystique dans les affaires humaines » ${ }^{1}$. Plusieurs éléments méritent d'être soulignés : l'individualisation du mort, son lien avec une relique, son influence sur les vivants, l'importance du rite et le rôle de la filiation ; éléments que l'on retrouvera chez certains Mayas des Basses Terres et qui seront confrontés avec ceux qui ont été décrits dans plusieurs sociétés africaines ou asiatiques.

Notre étude prendra appui autant sur des documents de l'époque coloniale, $\mathrm{du} \mathrm{XvI}^{\mathrm{e}}$ siècle, que sur l'ethnographie du $\mathrm{xx}^{\mathrm{e}}$ siècle. La question du culte des ancêtres doit en effet être abordée, dans le cas des Mayas, de manière diachronique. Les sources coloniales nous renseignent sur des pratiques qui peuvent être interprétées comme relevant d'un tel culte. Par ailleurs, certains rituels actuels semblent en être une forme adaptée. Nous constaterons que de nombreux rites, funéraires notamment, sont restés inchangés jusqu'à récemment et gardent un caractère maya malgré la conversion au christianisme. Nous pouvons aussi considérer l'étude du culte des ancêtres comme celle d'un processus de réinterprétation de la doctrine catholique espagnole par les Mayas des Basses Terres.

Enfin, nous adopterons un point de vue comparatif. Les Mayas des Basses Terres se divisent en quatre groupes qui appartiennent tous à la même famille linguistique dite "yucatèque » : 1) Les Mayas proprement yucatèques vivent dans les Etats actuels du Yucatán, du Campeche et du Quintana Roo, ainsi qu'au 


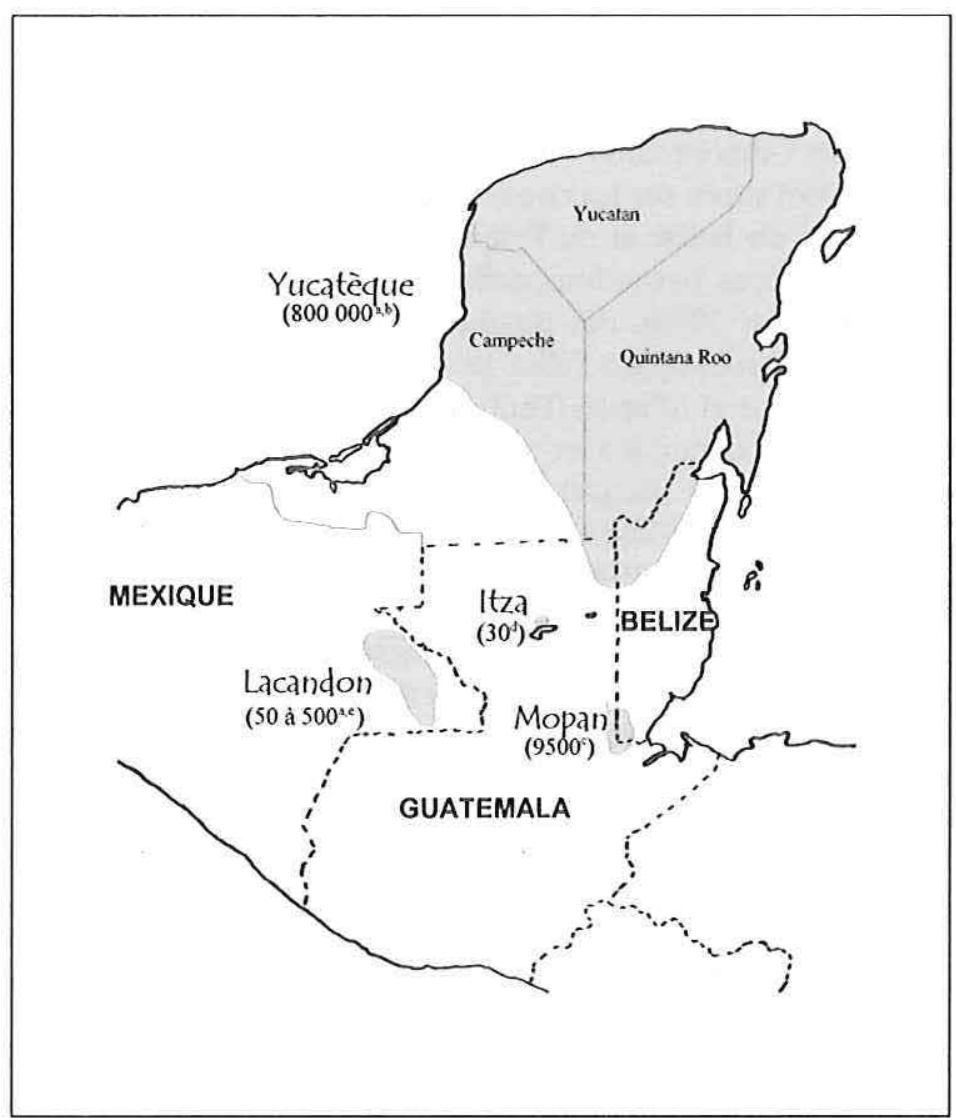

Répartition et nombre de locuteurs du groupe linguistique yucatèque

a : XII ${ }^{e}$ Censo general de la población y vivienda, Mexico, 2000

b : SIL, 1991

c: : SIL, 1990

d: V. Vapnarsky, communication personnelle

e : Nah bolom

nord du Belize. Ils furent convertis au catholicisme dès l'arrivée des Espagnols dans la péninsule au Xvi ${ }^{\mathrm{e}}$ siècle. Les Yucatèques du Quintana Roo sont en réalité originaires de l'État du Yucatán, mais ont migré dans la partie orientale de la péninsule suite à leur première défaite lors de la guerre des Castes (1847-1901). 2) Les Mayas-Itza' occupaient lors de la Conquête une vaste partie du Petén et, en particulier, les rives du lac Petén-Itza' (Guatemala actuel). Leur histoire est mal connue et encore sujette à controverses, mais ils sont des descendants des Mayas 
du Yucatán. Certains auteurs pensent qu'ils émigrèrent vers le sud dans le courant du XIII ${ }^{\mathrm{e}}$ siècle. Ils ont été les derniers à résister aux Espagnols, jusqu'à leur défaite finale en 1697, puis furent rapidement convertis par des frères franciscains. Leurs descendants vivent actuellement dans deux villages (San Andrés et San José) situés sur les rives du lac Petén-Itza'. 3) Les Mayas-Mopan vivent dans le sud du Belize et du Petén. Ils furent, eux aussi, convertis dès le $\mathrm{xvl}^{\mathrm{e}}$ siècle. 4) Les Mayas-Lacandons, enfin, seraient, selon certains auteurs (Boremanse 1998; De Vos 1988), des dissidents Itza' provenant de catégories de populations n'appartenant pas à l'élite. Ils se trouveraient dans le Petén depuis au moins le Postclassique et, d'après Thompson (1977), dans le bassin des fleuves Pasión et Usumacinta depuis le XvII ${ }^{\mathrm{e}}$ siècle. Nous diviserons ces quatre groupes en deux sous-ensembles. Le premier que nous nommons "yucatèque » réunit les Mayas-Yucatèques, les Mayas-Mopan et les Mayas-Itza' Le second est celui des Lacandons et présente certaines différences notables avec le premier. Il n'a jamais été vraiment évangélisé, malgré quelques tentatives avortées de missionnaires. De plus, les Lacandons ont, dans la mesure du possible, évité d'avoir des contacts avec les autres groupes, qu'ils soient mayas ou non. À travers notre étude des pratiques et des rites relatifs aux morts et au culte des ancêtres, nous essaierons de montrer en quoi ce contraste culturel entre ces deux sous-ensembles est pertinent.

Dans un premier temps, nous présenterons les sources utilisées. Puis, en suivant Fortes (1987, p. 72) : « si le culte des ancêtres est subsumé par le culte des morts, alors sa signification doit être recherchée dans les croyances et les pratiques coutumières concernant la mort, l'âme, les revenants, les esprits et l'au-delà » ${ }^{2}$, les rites funéraires et la notion d'âme chez les Mayas des Basses Terres devront être analysés. L'examen du traitement du corps et des os démontrera ensuite que ces derniers peuvent être considérés comme des reliques. Il faudra aussi interpréter l'importance de la nourriture dans les rites liés à la mort et au culte des ancêtres et s'intéresser à la maison, lieu à la fois de vie, de mort et de culte. Les rapports entre morts et vivants ne sauraient évidemment être omis, notamment lors du rite du hanal pixan. On esquissera finalement une comparaison entre les indices recueillis chez les Mayas et ce qui, dans les sociétés africaines et asiatiques, a été pris pour caractéristique du culte des ancêtres.

\section{LES SOURCES}

Ici nous convoquerons aussi bien des sources coloniales du XvI ${ }^{e}$ siècle qu'ethnographiques du $\mathrm{xx}^{\mathrm{e}}$ siècle. Cet écart de presque cinq siècles n'est pas totalement rédhibitoire, à voir la relative permanence qui existe entre les deux époques, en ce qui concerne au moins les coutumes funéraires. Certaines d'entre elles, décrites à l'époque coloniale, furent encore observées au début du siècle dernier. 
Notre principale source concernant la période coloniale est l'œuvre de Diego de Landa, Relación de las cosas de Yucatán (1985), datant de 1566. L'auteur y décrit les habitudes funéraires des habitants du nord de la péninsule et transcrit quelques-unes des idées des Mayas de l'époque sur la mort et l'après-vie. Il évoque certains usages qui, comme nous le montrerons, font penser à un culte des ancêtres ${ }^{3}$.

Si un regain d'intérêt pour les Mayas se manifeste dès la fin du XvIII ${ }^{\mathrm{e}}$ siècle, ce n'est qu'au début du $\mathrm{xx}^{\mathrm{e}}$ siècle qu'apparaissent les premiers écrits sérieux d'ethnographie. Les premiers mayanistes sont d'ailleurs davantage des historiens ou des archéologues et, dans leurs travaux, la partie consacrée aux Mayas contemporains jouxte bien souvent l'étude d'un site archéologique situé non loin du village ethnographié. C'est le cas par exemple de Thomas Gann (1918), qui a travaillé avec les Yucatèques de Santa Cruz (près du Río Hondo), ou de Thompson (1930), qui s'est intéressé aux Mopan du Belize actuel. Ce type d'approche traduit la volonté de l'époque, guidée par une idéologie de la permanence, de répondre aux questions posées par les ruines à l'aide d'éléments tirés de la mythologie ou de la religion des Mayas contemporains. Le travail un peu antérieur de Tozzer (1907) avait, lui aussi, consisté à rechercher chez les Mayas-Lacandons, par une comparaison avec les Mayas de la péninsule et ceux des Hautes Terres, des traces de la culture ou des croyances des anciens habitants des cités classiques. Tozzer, qui s'est rapidement rendu compte de l'impossibilité d'un tel projet, nous laisse un travail d'ethnographie comparée aussi précis que précieux.

La première réelle monographie sur les Mayas-Yucatèques paraît en 1934. Elle est le fruit d'une collaboration entre un ethnologue américain, Robert Redfield, et son assistant mexicain, alors instituteur du village, Alfonso Villa Rojas : Chan Kom, a maya village reste, aujourd'hui encore, la référence citée dans tous les travaux sur les Mayas du Yucatán. Une dizaine d'années plus tard est publié The Mayas of East Central Quintana Roo (1978), d'Alfonso Villa Rojas. Bâti sur le même schéma monographique que Chan Kom, cet ouvrage permet de mettre au jour les différences culturelles séparant les Mayas descendants des Cruzo'ob (les révoltés de la guerre des Castes qui auraient fui du Yucatán pour se réfugier dans la forêt du Quintana Roo), de ceux du nord de la péninsule. Si ces deux monographies font de nos jours encore autorité, elles ne sont pas uniquement à traiter comme des ouvrages ethnographiques mais aussi comme des sources d'ethnohistoire. En effet, la société maya a connu de profonds changements au cours des dernières années, autant dans certaines pratiques culturelles (influence de l'occidentalisation avec le développement de la scolarisation en espagnol, introduction de la télévision, etc.) que sur les plans économique (développement du tourisme et conséquences de la signature du traité de libre-échange ALENA en janvier 1994) et religieux (prosélytisme protestant) ${ }^{4}$.

Les travaux ethnologiques concernant les Lacandons, quant à eux, sont quasiment tombés dans le domaine historique (y compris les plus récents), 
notamment après la mort du vieux Chan K'in de Naha', informateur principal et détenteur presque exclusif de tout un pan du savoir lacandon, disparu avec lui. Les deux références auxquelles il faut avoir recours sont l'ouvrage de Tozzer (1907) et celui plus récent de Boremanse (1998). Ce dernier est une excellente monographie des deux groupes lacandons du nord et du sud, l'auteur n'hésitant pas à puiser dans les études précédentes lorsque les observations ne sont pas suffisantes. Bien que sa date de parution soit assez récente, les enquêtes de Boremanse remontent pour la plupart aux années 1970 et 1980.

Les données recueillies dans les études que l'on vient de citer seront complétées par quelques informations de terrain récoltées lors d'enquêtes effectuées dans le village maya de $\mathrm{x}-\mathrm{K}$ 'opch'en ${ }^{5}$, Quintana Roo. Ces dernières se sont déroulées de novembre 2002 à février 2003, puis de mai à août 2003. Pendant ces séjours, nous avons pu assister aux rituels effectués en l'honneur des morts, qui débutent le $1^{\text {er }}$ novembre et s'achèvent le 24 décembre.

\section{LE DÉCÈs, LA MORT ET LA NOTION D'ÂME}

Avant d'aborder le culte des ancêtres proprement dit, il n'est pas inutile de s'intéresser aux idées des Mayas concernant la mort et l'après-vie et, naturellement, aux rites funéraires, ainsi qu'aux relations qu'entretiennent vivants et morts durant cette période délicate qu'est le décès d'un membre de la communauté.

\subsection{Les rites funéraires}

La mort est rarement conçue par les Mayas comme relevant seulement de causes biologiques. Elle est avant tout décidée par Dieu (Villa Rojas 1978, p. 423). En outre, si le décès a une origine avérée (maladie, accident, etc.), il n'en a pas moins aussi des causes indirectes, impliquant parfois tout l'entourage de la personne. Lorsqu'une personne âgée vient à disparaître, il est rare que la cause de sa mort soit attribuée uniquement à son grand âge. Burns (1993), rapportant le décès d'un de ses amis yucatèques, raconte comment chacun au village avance sa propre théorie, allant du suicide jusqu'au complot du beau-père.

La mort est plutôt conçue comme un départ, celui de l'âme (pixan). Redfield et Villa Rojas (1934) rapportent que l'âme d'un mourant sort de son corps pour effectuer un premier voyage vers le purgatoire et la Gloria (le paradis maya). Le départ définitif de l'âme est un moment douloureux et délicat. Forcément pécheresse, elle répugne à quitter le corps, de crainte d'être enlevée par les démons grimaçants et hurlants, les okol pixan ou " voleurs d'âmes » (Villa Rojas 1978), qui entourent le défunt. Selon Redfield et Villa Rojas (1934), ces derniers sont originaires du Metnal (lieu de l'inframonde, correspondant aujourd'hui à l'enfer 
maya). Afin de les éloigner du mourant, le maestro cantor, représentant religieux catholique, récite des prières (ibid.). Au Quintana Roo, quand la douleur devient trop intense et que les prières ne suflisent plus, on fouette doucement la personne avec une corde afin d'alléger, par cette sorte d'expiation, le poids de ses péchés (Villa Rojas 1978). L'évêque Diego de Landa, évangélisateur du XvI ${ }^{\mathrm{e}}$ siècle, parle également de démons qui viennent chercher l'âme du disparu. On peut se demander s'ils ne sont pas les okol pixan, les voleurs d'âmes des Yucatèques actuels. Ils sont envoyés par Hunhau, le maître du Metnal. Tozzer (1941, p. 132, note 618) précise en note du texte de Landa que ce type de démon est aussi connu sous les noms de Kisin (un terme encore employé chez les Yucatèques, les Itza' et les Lacandons) et Yum Kimil ${ }^{6}$ (signifiant « seigneur de la mort » et toujours usité dans cette acception chez certains Yucatèques).

Chez les Lacandons, le mourant est étendu dans son hamac. Une attitude délirante est interprétée comme le signe que l'âme ou pixan a déjà quitté le corps. Un rite est donc effectué pour protéger les encensoirs ${ }^{7}$ du fantôme du défunt, qui rôde sur terre pendant les jours suivant son décès (Boremanse 1998).

Les Mayas-Yucatèques ne laissent jamais la famille assurer la préparation du cadavre. Ils craignent en effet que l'âme du mort n'emporte avec elle un proche vers l'au-delà. Quelques voisins et amis, du même sexe que le défunt, se chargent donc de venir dans sa maison pour le préparer. Ses habits sont changés et il est enveloppé dans un linceul, puis placé sur une table ou parfois, comme au Quintana Roo, sur des palmes de guano ${ }^{8}$. Au Yucatán, on allume deux bougies à chaque extrémité de la table. À la taille du défunt est placée une ceinture de coton, composée de cinquante nœuds (correspondant au nombre d'ave maria dans le rosaire), avec laquelle, dit-on, l'âme sera fouettée devant Dieu pour expier ses péchés. Au Quintana Roo, des bougies sont pareillement placées de chaque côté de la tête. On installe aussi une table sur laquelle on dispose une petite croix, une bougie et cinq ou sept calebasses d'atole (boisson à base de maïs), on peut $\mathrm{y}$ ajouter de la nourriture et des tortillas. La veillée, $p^{\prime} i x$ ich, commence et dure vingt-quatre heures. Redfield et Villa Rojas expliquent qu'au Yucatán, alors que les jeunes jouent aux cartes, se racontent des histoires et boivent de l'atole et de l'alcool, les plus âgés récitent des prières. Au Quintana Roo, pendant que les hommes fument, boivent et que les femmes s'affairent à des travaux de cuisine, un prieur spécialisé énonce les oraisons. Le lendemain on conduira le corps au cimetière où il sera inhumé (Redfield et Villa Rojas 1934; Villa Rojas 1978).

Chez les Lacandons, au contraire, c'est un membre de la famille qui se charge de préparer le cadavre. Il est vrai que le mode de résidence en famille restreinte (le mari, ses femmes et ses enfants) ne facilite pas l'intervention d'autres personnes. On place dans les mains du défunt un épi de maïs, un os de singe ou de pécari et une mèche de cheveux. Ces éléments seront utiles à l'âme pour se débarrasser des animaux (poules, chiens et poux... ${ }^{9}$ ) qui l'attaqueront successivement sur le sentier menant au royaume des morts. Sous le cadavre, on place une coupelle en 
terre cuite contenant des braises pour le garder chaud, sinon, le mort pourrait aller se plaindre aux dieux et accuser ses parents de l'avoir laissé refroidir. Les dieux puniraient alors cette négligence, en faisant mourir une autre personne de la famille. Il en va de même si des braises trop ardentes brûlent le corps. Le lendemain de la mort, le cadavre sera conduit à sa dernière demeure, une tombe dans la forêt (Boremanse 1998).

Notons dès à présent l'importance de la nourriture dans les rituels funéraires yucatèques comme lacandons. Les différences principales entre les deux groupes résident essentiellement dans la limitation du groupe social lors des cérémonies. Chez les Lacandons, seuls les membres de la famille sont invités à participer aux funérailles alors que, pour les Yucatèques, des personnes extérieures à l'unité familiale sont requises, en particulier pour la préparation du cadavre.

\subsection{La période liminale}

Après le décès d'une personne, il existe une période liminale, plus ou moins longue, pendant laquelle l'âme (ou les âmes) du mort erre(nt) parmi les vivants.

Les Yucatèques pensent que les âmes des défunts peuvent être vues fréquemment par les animaux, au contraire des hommes qui ne les perçoivent qu'occasionnellement. On dit notamment que, lorsqu'un chien hurle toute la nuit ou qu'un animal grogne avec effroi, c'est le signe d'une mort prochaine (Redfield et Villa Rojas 1934). Cela semble confirmer la croyance selon laquelle l'âme se détache du corps avant que la mort ne frappe.

Le troisième jour après le décès est d'une grande importance dans toutes les Basses Terres. En effet, c'est à ce moment qu'est révélé au défunt son nouvel état. Alors que le mort yucatèque continuait de vivre normalement parmi les siens sans s'être rendu compte de rien, il entend son nom lors de l'office du troisième jour de sa mort célébré dans sa maison. Cela le plonge dans une profonde amertume et il décide alors de retourner jusqu'à sa tombe, où il reste toute la nuit à pleurer. On peut d'ailleurs parfois entendre ses lamentations. À l'aube, il commencera son voyage vers son lieu de vie future (Metnal ou Gloria) (Villa Rojas 1978). Il en va de même à $\mathrm{x}-\mathrm{K}$ 'opch'en et l'on dit aussi qu'un aïeul du défunt se présente à lui pour le guider dans l'après-vie.

Au Yucatán, l'âme retourne dans sa maison jusqu'au septième jour. Durant cette intervalle la maison ne doit pas être nettoyée car le mort revient y récupérer tout ce qui lui appartient et rassembler ses péchés qu'il emportera au Paradis pour être jugé (Redfield et Villa Rojas 1934, p. 201). Thompson (1930) aussi mentionne une période de huit jours pendant laquelle l'esprit séjourne sur terre et peut apparaître aux vivants. Les âmes de ceux qui ont péri de male mort (accident, meurtre, etc.) sont condamnées à rester sur le lieu de leur trépas. Cette conception, fait remarquer Ruz (2003), est proche de celle dite du susto (notion liée en partie à la peur et à la violence d'un choc), c'est-à-dire la perte d'une partie de 
l'âme lors d'une chute par exemple. Certaines âmes pécheresses peuvent ainsi être enfermées dans des rochers ou coincées dans des arbres (d'où le grincement de certains arbres lorsqu'il y a du vent). Elles restent là jusqu'à ce que quelqu'un les délivre de leur prison minérale ou végétale (en soulevant une pierre par exemple) et leur permette de rejoindre la Gloria.

Pour les Lacandons du nord, Boremanse (1986, p. 73 ; 1998) précise qu'un homme mort n'est pas conscient de son état. Il arrive au ciel chez les dieux et une déesse mineure, qui ressemble à sa femme, vient lui servir du balche' ${ }^{10}$. Le lendemain, il rappelle celle qu'il croit être sa femme et c'est à ce moment qu'elle lui révèle qu'il est mort. Cela provoque dans l'âme du défunt une grande tristesse, elle quitte alors le monde céleste de Hach Ak Yum ${ }^{11}$ et descend dans l'inframonde.

Il est notable, pour les Yucatèques comme pour les Lacandons, que l'âme du disparu séjourne sur terre pendant une période déterminée. Ainsi le décès et la mort seraient à différencier, la mort physique n'était qu'une étape de la mort. Le processus débute donc par le départ de l'âme du corps, se poursuit par le décès de l'enveloppe corporelle et, enfin, après une période liminale, par la migration de l'âme vers la Gloria ou le Metnal. Comme le remarque déjà Hertz en 1907, la période liminale est celle du deuil pour les vivants dont les sentiments vis-à-vis du mort sont ambivalents, mêlant crainte et commisération. C'est aussi le temps pour le mort de faire le deuil de sa vie perdue.

\subsection{La notion d'âme ou pixan}

Si nous avons jusqu'ici employé le terme d'«âme» pour la simplicité de l'exposé, il est nécessaire d'en donner une description précise. Les Mayas, bien que convertis au christianisme pour la plupart, ne considèrent pas l'âme dans un sens chrétien.

Chez les Lacandons septentrionaux, il existe trois parties différentes qui composent l'individu : le pixan, siégeant dans les poumons, peut être considéré comme « l'âme »; le kisnin, " les veines des bras et des jambes », a souvent le sens de « spectre »; le sol, « l'enveloppe », renvoie au corps. Dans le rêve et lors de la mort, le pixan sort du corps tout en conservant exactement son image; selon Boremanse (1998, p. 91), il devient le double du corps. Le cœur est dit ne chi'k yum in pixan, « le grand seigneur de mon pixan » et les poumons hach in pixan, « mon vrai pixan ». Le corps est qualifié de $u$ nah winklil : "son grand propriétaire/ possesseur » (de nah " grand " et u winklil, "son propriétaire »). Selon Bruce (1968, p. 33), ce dernier terme correspondrait morphologiquement à wink-il-il, probablement dérivé du terme winik (" personne »). Cette expression désigne le corps après que l'âme l'a quitté (le corps mortel dont la chair va pourrir). Un autre nom pour le corps est sol, qui renvoie aussi à l'écorce des arbres. Au moment de la mort, les Lacandons disent généralement $t u$ sol-in-t-ah $u$ bäh, expression utilisée pour désigner la mue du serpent et qui signifie littéralement $:$ «il ou elle a 
enlevé sa peau/son enveloppe ". De même, lorsque quelqu'un est sur le point de trépasser dans son hamac, certains disent que seul son sol (Boremanse le traduit par covering, "couverture ») est là, alors que son pixan est déjà parti (ibid.). Notons que les Lacandons du nord pensent qu'il existe une seconde âme ( $k a$ ' pixan) vivant dans l'inframonde sous la forme d'un singe-araignée. Si elle est tuée comme gibier par Kisin ${ }^{12}$, le seigneur de l'inframonde (Metnal), l'individu dont elle est le double meurt également.

Pour les Lacandons méridionaux, lors de la mort, l'âme se sépare en deux entités dont la principale est $i k$ hach pixan, "notre vraie âme ", ou $i k$ hach winkiil, « notre vrai propriétaire/possesseur ». Pendant le sommeil, l'âme est un double qui erre hors du corps et, au moment de la mort, elle rejoint les dieux. L'autre entité est une petite partie du cœur correspondant, semble-t-il, à l'aorte. Cette part de l'âme, qui se rend chez Kisin pour brûler dans le Metnal, est connue sous le nom de $u$ chu'uch ik pixan, "le sein de notre âme », ou bien $u$ wich ik pixan, « l'œil de notre âme ». Le corps semble être lié aux palmes de guano, un matériau couramment utilisé dans la vie quotidienne, car il est dit $i k x a^{\prime} n$, « nos palmes de guano " ${ }^{13}$. Un mythe lacandon raconte qu'un dieu, pour échapper à ses agresseurs, fit un double de lui-même avec des palmes de guano (ibid., pp. 371-374). $X a^{\prime} n$ désigne aussi les pierres placées dans les encensoirs, parfois nommées $u b a ̈ k e l$ $k^{\prime} u h$, « le corps des dieux ». Les Lacandons méridionaux pensent qu'ils ont un compagnon ( $u$ wet man, littéralement « celui qui va avec ») qui les suit partout. C'est pour cette raison, disaient les anciens, qu'il fallait jeter un peu de nourriture lorsqu'on prenait un repas dans la forêt. Ik kisin, " notre spectre », correspond au spectre des Lacandons septentrionaux.

Ainsi, chez les Lacandons, on peut distinguer dans trois parties l'individu : 1) l'âme, ou pixan pour les Lacandons du nord et $i k$ hach pixan (" notre vraie âme ») à laquelle il faut ajouter " le sein de l'âme " (u chu'uch ik pixan) pour ceux du sud ; 2) le corps qui est entendu comme une enveloppe pour les Lacandons septentrionaux, vraisemblablement faite de palmes pour les méridionaux ; 3) le spectre (kisnin ou $i k$ kisin) dont on ne sait que peu de chose. Il semble que cette autre âme soit une sorte de fantôme rôdant autour de son ancienne demeure qui lui manque et qui tente d'effrayer les vivants (Boremanse 1998 ; Tozzer 1907). Pareille division existe au moins en partie chez les Mayas de la péninsule, même si elle est plus subtile à discerner. La notion de pixan est traduite dans les dictionnaires coloniaux par " âme », mais désigne aussi « le pouls », « la pulsation », « le battement de cour ». Cette seconde acception existe aussi en lacandon. Dans le dictionnaire de Bricker et al. (1998), pixàan traduit l'«âme" et l'on peut trouver comme définition de pix : couvrir, envelopper, emballer; quant à a'an, c'est un résultatif qui marque souvent un participe (par exemple, $t s^{\prime} o k$-ă'an : " fini », " terminé »). Ainsi, l'âme des Yucatèques serait une entité (essence vitale ?) qui est « recouverte ». Le corps se dit uwíinkil, terme qui peut être traduit par «son corps » mais aussi « son armature », «sa structure » (Bastarrachea 
1992). On constate donc que, au moins dans le vocabulaire, le corps et l'âme sont complémentaires et associés. Le corps, considéré comme une structure, paraît être le siège du pixan puisqu'il le recouvre tout au long de la vie. Quant au spectre, il ne semble pas exister chez les Mayas-Yucatèques.

D'un autre côté on doit ajouter que l'âme (pixan) est liée au cœur. Ce dernier est aussi le siège des émotions chez les Yucatèques, il est dit óol, "cœur ", " désir », " esprit ». Ce terme ne se réfère pas à l'organe - qui se dit puksi'ik'al mais bien à une image figurée du siège des émotions. D'ailleurs, la plupart des expressions se rapportant aux émotions sont des composées de óol. Citons quelques exemples : ok'om-'óol « la tristesse », há'as-'óol « la peur », má'a-'óol "le manque d'énergie ou la timidité » (Bricker et al. 1998). La relation entre âme(s) et cœur existe aussi dans d'autres groupes mayas, en particulier les Tzeltal (Pitarch 1996; Figuerola 2000). Chez les Lacandons, la relation est si forte que le même terme (pixan) désigne le cœur et l'âme. Cette dernière, au moins linguistiquement, est bien séparée du corps, considéré comme son enveloppe, en particulier lorsque les Lacandons nomment le corps sol, l'« écorce ». Lors de la mort, le corps reste sur terre (et même dans la terre). Il arrive d'ailleurs qu'au Quintana Roo, dans certains contextes rituels, l'âme, pixan, soit opposée au terme lu'um qui signifie plus généralement «terre" (Vapnarsky 2000, p. 460 note 28). Si les Lacandons considèrent le corps comme la partie périssable de l'individu, nous verrons qu'il est d'une importance considérable pour les Yucatèques (notamment pour le rite du hanal pixan) qui prennent soin de le garder près de leur habitation.

Pour les Mayas-Yucatèques et pour les Lacandons, le corps est donc considéré comme une enveloppe destinée à couvrir l'âme (ou les âmes dans le cas des seconds). La mort engendre la séparation de toutes ces composantes de l'individu et, si le corps reste sur terre, les âmes vont dans des lieux qui peuvent varier selon l'âge au moment de la mort ou selon les péchés commis durant la vie. Les enfants morts jeunes sont considérés comme n'étant pas encore souillés ${ }^{14}$ et se dirigent vers le paradis : la Gloria pour les Yucatèques, chez Hach Ak Yum, au ciel, pour les Lacandons. Les pécheurs yucatèques voient leur âme partir vers l'enfer ou Metnal.

\section{LE TRAITEMENT DU CORPS ET DES OS}

Pour les Lacandons, le corps n'est qu'une enveloppe qui pourrit après le décès, soulignant ainsi le caractère contingent de l'homme. La personne récemment décédée pleure sur sa nouvelle condition (désormais une âme) désirant retourner sur terre parmi les siens ; mais afin de l'en dissuader, un dieu mineur l'accompagne jusqu'à sa tombe pour lui montrer sa dépouille corrompue. Face au constat du pitoyable état de son cadavre en putréfaction, elle est tellement dégoûtée qu'elle ne cherche plus à rejoindre son corps et perd ainsi la nostalgie de la vie 
terrestre (Boremanse 1986, pp. 75-76; 1998). L'âme n'a alors plus de relation avec le corps qu'elle a définitivement abandonné.

Il en va tout autrement pour les Yucatèques, en particulier à l'époque coloniale et préhispanique. Dans sa Relación, Landa, décrivant des pratiques funéraires relatives aux personnes de haut rang, souligne l'importance de ce que, à l'instar de McAnany (1995), nous considérons comme des reliques :

Pour les personnes de grande estime, ils brûlaient leur corps et plaçaient les cendres dans de grandes urnes et ils construisaient des temples par-dessus [...]. On a découvert que, quand ils étaient d'un rang très élevé, ils enfermaient leurs cendres dans des statues de poteries creuses. Le reste des gens de haut rang faisait pour leurs pères des statues de bois où un creux était laissé à l'arrière de la tête, puis ils brûlaient une partie du corps et plaçaient là les cendres et le refermaient ; ensuite ils décollaient le cuir à partir de la base du crâne et le collaient là, ils enterraient les restes du corps comme ils en avaient l'habitude; ils gardaient ces statues avec le plus grand respect parmi leurs idoles ${ }^{15}$.

On constate que, malgré quelques différences de traitement selon le rang social, le but de ces pratiques est de constituer des reliques afin de pouvoir leur rendre un culte. On peut se demander si ces statues ou ces urnes étaient individualisées ou si elles ressemblaient au défunt. Un autre passage de la Relación, relative aux Cocom (une grande lignée de dirigeants de l'époque) et aux usages réservés aux rois, nous éclaire sur ce point :

Ils avaient coupé la tête des seigneurs Cocom au moment de leur mort ; après les avoir cuites, ils retirèrent la chair puis scièrent la partie arrière du crâne, laissant la partie avant avec la mâchoire et les dents. Une sorte de béton vint remplacer la chair sur ces moitiés de crâne, rendant à la perfection ce à quoi ils ressemblaient. Ils les placèrent avec les statues et les cendres, toutes étaient ainsi gardées sur les oratoires des maisons avec grand respect et égard ${ }^{16}$.

Pour les seigneurs de plus haut rang, la volonté de faire des reliques ressemblantes est évidente. Mais ce ne sont pas là simplement des supports de la mémoire ; ces reliques étaient vénérées et on leur faisait même des offrandes de nourriture lors des banquets afin de contenter leurs âmes (Tozzer 1941, p. 131) ${ }^{17}$. Il apparaît donc que l'âme est toujours liée aux restes corporels et, du même coup, ces reliques sont un moyen d'entrer en contact avec le mort.

À propos des décapitations, Avendaño (Roys 1943, p. 27) rapporte que les Cehaches et les Itza' du lac Petén avaient pour habitude de décapiter les personnes les plus âgées (vers cinquante ans) pour éviter qu'elles ne deviennent des sorciers et donc dangereuses. Les prêtres qui gardaient les idoles étaient épargnés car on avait pour eux un grand respect. Il n'est pas précisé si les crânes étaient conservés, on peut toutefois le penser si l'on se réfère à la pratique des Itza' modernes. Les habitants du lac Petén-Itza' sont réputés pour pratiquer, le $1^{\mathrm{er}}$ novembre, un rituel unique dans la péninsule : lors du hanal pixan, une procession vient apporter, chez 
les villageois qui en ont fait la demande, un des trois crânes habituellement gardés dans l'église. Des offrandes de nourriture et de boisson lui sont faites sur l'autel familial où il est déposé pour un moment, avant de repartir chez d'autres personnes et de retourner enfin à l'église. Chacun des crânes possède sur le front une marque géométrique en gomme qui n'est pas sans rappeler la description de Landa de crânes recouverts de bitume. Les habitants disent qu'ils sont ici depuis des temps immémoriaux et qu'ils appartenaient à des gentes muy finas (des « gens de grande qualité ») ou des priostes (des prêtres). Doit-on en conclure que cette pratique est un héritage des temps préhispaniques ? Même si l'origine des crânes n'est pas certaine, les habitants considèrent qu'ils furent des gens très importants et le fait qu'ils aient été humains les place dans une lignée en relation directe avec les habitants actuels, à la différence des saints, comme en témoigne un Itza' : « Ils sont plus importants que les saints car ces derniers sont faits de bois. Les crânes ont été des êtres humains et ont été vivants. Ce sont de vrais os tels que nous en avons ${ }^{18}$. Il est diffícile de dire ce que représentent ces crânes pour les habitants et pourquoi ils les accueillent et leur font des offrandes dans leur maison. Sont-ils considérés comme des ancêtres ? Quel est leur lien de filiation avec les habitants de la communauté ? En sont-ils des représentants? Aucune source ethnohistorique ou ethnologique ne peut malheureusement répondre aujourd'hui à ces interrogations. Cependant, il est manifeste que l'aspect communautaire et la relation avec les morts sont liés et soulignés par ce rituel.

Au Yucatán, la période du hanal pixan est propice à une cérémonie qu'on ne retrouve nulle part ailleurs. Il s'agit d'exhumer les ossements des morts inhumés deux ou trois années auparavant. Les sols peu profonds et secs de cette région facilitent cette pratique qui donne lieu à une cérémonie comparable à une veillée funéraire. Les os déterrés au cimetière sont bénis et placés dans une petite boîte qu'on emportera dans la maison de la famille. Après plusieurs cérémonies où l'on appelle le mort par son nom, les restes du défunt sont rapportés au cimetière où ils demeureront. Ne pourrait-on pas voir dans cette cérémonie une lointaine réminiscence du rite, décrit par Landa au $\mathrm{XvI}^{\mathrm{e}}$ siècle, au moment où les cendres des dignitaires étaient placées dans des urnes que l'on vénérait à chaque grande fête communautaire? La crémation étant prohibée par l'Église, les Mayas attendraient une décomposition naturelle du corps pour pouvoir enfin achever le rite funéraire. Redfield et Villa Rojas (1934) proposent, quant à eux, une explication utilitaire de cette pratique : elle servirait à libérer de l'espace dans les cimetières. On ne retrouve pas cette cérémonie au Quintana Roo où vivent pourtant des MayasYucatèques qui s'y sont installés après la guerre des Castes. Peut-être la composition du sol, plus humide qu'au Yucatán, gêne-t-elle cette pratique ? Cette cérémonie n'est pas sans rappeler les doubles obsèques pratiquées par de nombreux groupes (Hertz 1970) et dont le but est de faire accéder le mort au statut d'ancêtre.

Nous le voyons, si, pour les Lacandons, le corps n'a plus d'importance après la mort, il est, pour les Yucatèques, un moyen d'entrer en contact avec l'âme qui 
lui reste liée. Ainsi, les reliques décrites par Landa opèrent comme intermédiaires pour rendre un culte à l'âme d'une personne bien individualisée. À x-K'opch'en, on nous a confié que les âmes des morts, lorsqu'elles redescendent sur terre, arrivent d'abord à l'endroit où se trouvent leurs os avant de se diriger vers le lieu où on les appelle (l'église ou la maison familiale). Les ossements semblent donc agir comme un repère essentiel dans l'espace du mort, un point de passage entre l'univers des âmes et celui des vivants.

\section{L'IMPORTANCE DE LA NOURRITURE}

La nourriture est omniprésente dans de nombreux rituels yucatèques et lacandons, en particulier lors des cérémonies funéraires. Mais son utilisation n'est pas restreinte à l'autel ou à l'oratoire et on la retrouve aussi enterrée avec le défunt.

À l'époque coloniale, Landa (Tozzer 1941) rapporte à propos des cadavres que les Mayas « les mettent dans un linceul, remplissant leur bouche avec de la pâte de maïs, qui est leur nourriture et leur boisson qu'ils nomment koyem $"{ }^{19}$. Dans les années 1930, Thompson note que, dans la région actuelle du Belize, les cérémonies funéraires sont fort simples car le corps est enterré le plus rapidement possible sans prières. Il souligne que « des possessions personnelles et de la nourriture étaient inhumées avec [le corps], mais [que] cette coutume disparaît progressivement ${ }^{20}$. Il est aussi courant que, le troisième et le huitième jours après le décès, les proches du défunt se rendent en forêt afin d'y « jeter un peu de

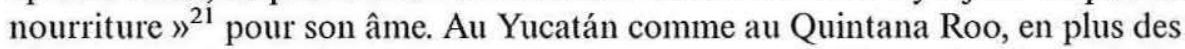
vêtements et de certains objets appartenant au mort, on dépose dans la tombe la calebasse dans laquelle il buvait (Redfield et Villa Rojas 1934 ; Villa Rojas 1978).

Chez les Lacandons, un filet - contenant une gourde de gruau de maïs, quelques tortillas, des bougies et des brindilles pour faire du feu - est disposé dans la tombe sur les genoux du mort. Les tortillas et le gruau sont offerts pour le voyage ; les bougies et les brindilles, pour les nuits solitaires dans l'inframonde. Des sources indiquent que la gourde de balche' placée près du corps serait une offrande pour Sukunkyum ${ }^{22}$ (Bruce et al. 1971, p. 123 ; Baer et Baer 1952, p. 241 cité dans Boremanse 1998). Durant les trois jours qui lui sont nécessaires pour rejoindre le royaume de Mensabäk $^{23}$, l'âme séjourne sur terre. C'est pourquoi de la nourriture est apportée quotidiennement sur sa tombe et le feu ravivé. Ces trois jours sont considérés comme une période délicate, pendant laquelle l'âme est susceptible d'apparaître aux vivants, en particulier lorsqu'ils replacent des offrandes sur la sépulture. Une telle rencontre peut être fatale. Les tortillas et le gruau sont ensuite rapportés à la maison et consommés par la famille car on dit que les âmes ne mangent que le pixan des aliments. Les Lacandons s'attendent cependant à ce qu'un peu de nourriture soit entamé (la moitié d'une tortilla, une gorgée de 
gruau). Si rien n'a été touché pendant les trois jours, alors il faudra en conclure que l'âme, trop pécheresse, ne reviendra jamais du Metlan (lieux des morts) où elle a été brûlée entièrement par Kisin (Boremanse 1998).

On constate chez les Lacandons et les Yucatèques, particulièrement ceux de l'époque coloniale, la volonté de nourrir l'âme par l'intermédiaire du corps. Ces pratiques qui consistent à placer des aliments près de la dépouille soulignent la corrélation entre les deux entités et renforcent notre thèse du corps conçu comme une relique, pont entre les vivants et l'âme.

Si la nourriture constitue, nous venons de le voir, une offrande essentielle, elle représente par ailleurs une limitation du groupe social, notamment à travers le lien de commensalité. C'est ce que fait remarquer Farriss (1984) quand elle écrit que « pour les Mayas de l'époque coloniale, la nourriture jouait un rôle majeur dans toutes les célébrations solennelles. Les banquets communs accompagnaient toutes les fêtes, les veillées et les autres célébrations pour les morts, la cérémonie de la pluie Cha-Chaac et, de façon plus apparente, toutes les occasions séculaires telles que les mariages ou les restaurations de toits. La commensalité sert clairement des intentions sociales. Les repas partagés établissent les limites d'un groupe, expriment son identité collective et renforcent sa cohésion interne ${ }^{24}$. On trouve toujours des témoignages de cette restriction du groupe social à travers le partage de nourriture dans les pratiques des Yucatèques ou des Lacandons. Ainsi, Boremanse (1998) raconte qu'une des difficultés de son enquête de terrain fut sa mise à l'écart systématique de la communauté lors des repas qu'il était forcé de prendre seul. Lors des cérémonies familiales pour les morts (hanal pixan ou autres), le partage de la nourriture suit des règles précises; seuls les parents et quelques amis sont autorisés à consommer les offrandes. Il en va de même pour les âmes dont l'accès à l'autel familial et au pixan des aliments est limité.

\section{LA MAISON : LIEU DE VIE, DE MORT ET DE MÉMOIRE}

Nous entendons ici la maison dans le sens d'unité d'habitation familiale qui rassemble, souvent chez les Mayas, plusieurs structures dans un périmètre de taille variable selon les groupes. Chez les Yucatèques, l'aire d'habitation, nommée sòolar, est généralement délimitée par des murets de pierre. À l'intérieur, peuvent vivre jusqu'à trois ou quatre familles nucléaires, chacune dans une demeure particulière. La cuisine est souvent commune et l'on note parfois l'existence d'un oratoire familial. Le type privilégié de résidence est patrilocal ; cependant, l'habitat a tendance à devenir simplement virilocal, voire néolocal. Dans le cas des Lacandons, les individus, dont le nombre peut varier de six à une quinzaine, résident tous dans l'unité d'habitation. Autour du chef de famille, souvent le membre le plus âgé et le plus respecté, vivent ses femmes et ses enfants. 
La maison, lieu de vie quotidienne, n'exclut pas la mort, bien au contraire. Dans la mesure du possible, les Yucatèques et les Lacandons souhaitent mourir chez eux, lieu normal du départ de l'âme. Pour cette raison, les Yucatèques pratiquent une ouverture dans le toit de palme au-dessus du hamac (ou du lit) du mourant; les anciens conseillent, pour les maisons modernes, de laisser une fenêtre près du toit (Redfield et Villa Rojas 1934 ; Villa Rojas 1978). Nous avons également constaté que la préparation du corps et sa veillée se déroulent dans la demeure du défunt. La maison est également un lieu de mémoire. C'est principalement là que l'on célèbre les rituels du jour des morts (hanal pixan) et où reviennent les âmes.

C'était aussi l'endroit légitime d'inhumation des morts. Les fouilles archéologiques ont montré que cette pratique était très générale avant la Conquête (McAnany 1995) et le récit de Diego de Landa atteste cet usage au Xvi ${ }^{\mathrm{e}}$ siècle en remarquant qu' « ils les enterraient dans ou à l'arrière de leur maison » ${ }^{25}$. Tozzer (1941) précise que Landa évoque là une pratique des gens du commun. Cette tradition était si fortement ancrée chez les Mayas que, malgré l'évangélisation et la conquête espagnole, Thompson peut écrire en 1930, à propos des Mopan du Belize, qu'« il y a encore trente ou quarante ans, les gens étaient souvent enterrés sous le sol de leur maison " ${ }^{26}$. Dans les mêmes années, Pacheco Cruz (1934, p. 32) rapporte, lui aussi, qu'au Quintana Roo les morts sont toujours enterrés peu profondément, sans cercueil, dans les maisons, les rues ou les places publiques. Il note aussi qu'« en d'autres communes ils vont même jusqu'à utiliser les églises ${ }^{27}$. De nos jours encore au Quintana Roo, les gens disent qu'ils ne voient pas d'obstacle à enterrer leurs proches dans la cour de la maison - ainsi qu'en témoigne l'existence de tombes datant d'une quinzaine d'années - mais qu'il est désormais plus pratique d'aller au cimetière.

La colonisation espagnole, l'évangélisation et, plus tard, les lois sur l'hygiène ont contraint les Mayas à inhumer, la plupart du temps, les morts dans le cimetière du village. La maison n'est donc plus le lieu unique de la mort; le cimetière impose désormais un détour obligé aux vivants comme aux morts. C'est en tous cas ce que l'on peut inférer de certaines pratiques comme celle qui consiste, le jour de l'enterrement, à disposer, sur le chemin menant de la maison au cimetière, des grains de maïs afin de s'assurer que l'âme du défunt trouve bien la tombe dans laquelle va désormais reposer son corps. C'est pour la même raison que les gens du cortège n'hésitent pas à crier le nom de la personne. De même, lors du hanal pixan, des bougies déposées sur les murets des maisons sont censées guider les âmes des enfants dans l'obscurité (Redfield et Villa Rojas 1934 ; Villa Rojas 1978).

Les Lacandons n'enterrent pas leurs morts sous leur maison mais dans les bois, à quelques centaines de mètres des habitations. Ce n'est pas à strictement parler un cimetière car les tombes végétales (faites de branches, de palmes sur un monticule de terre, etc.) sont rapidement absorbées par l'épaisse forêt du Chiapas. 
Toutefois, si les Lacandons n'ignorent pas où se situent les sépultures, ils ne s'y rendent que durant les quelques jours après le décès (Boremanse, communication personnelle). Pour éviter que l'âme ne revienne, on dépose des piments grillés (censés faire fuir âmes et démons ${ }^{28}$ ) sur les sentiers menant à son ancienne demeure ; on retrouve parfois cette pratique chez les Yucatèques.

Il se dégage clairement la volonté de la part des Mayas de considérer le lieu d'inhumation comme "la maison du mort», selon les termes utilisés par un habitant de $\mathrm{x}$-K'opch'en. Cette intention est évidente chez les Lacandons qui construisent une structure pareille à une maison mais dont les dimensions (1 à $1,5 \mathrm{~m}$ de haut) et la fonction sont réduites. Cet abri ne servira à l'âme que quelques jours, pour s'abriter et se restaurer (Boremanse 1998, p. 93). On retrouve cette même idée chez les Mayas-Tzotzil du Chiapas qui édifient un toit en chaume au-dessus de la tombe qu'ils considèrent alors comme la maison du mort (Vogt 1969, p. 220). Les K'iche' des Hautes Terres du Guatemala construisent des autels dont, selon Carmack, « le plus important est le warabal ja (maison où l'on dort) pour les ancêtres " ${ }^{29}$. Les Yucatèques aussi, au moment de creuser la tombe, édifient une petite structure de palme (Redfield et Villa Rojas 1934).

Toutefois, tous les morts ne bénéficient pas du même traitement. Le rang social du défunt peut déterminer son lieu d'inhumation. Ainsi, au Xvi ${ }^{\mathrm{e}}$ siècle, Landa rapporte que l'on construisait un petit temple où étaient placées les idoles contenant les cendres des dignitaires ${ }^{30}$ (Tozzer 1941, p. 130). Pacheco Cruz (1947, p. 28) a observé, dans les années 1940, au Quintana Roo, l'enterrement d'un chef de village (el Jefe de Tribu) dans l'école. Les habitants, « de par son investiture, le considéraient digne de pouvoir se perpétuer dans un tel lieu et non dans la rue ${ }^{31}$. Ces exemples, qui restent malgré tout assez rares, soulignent une certaine hiérarchie entre les morts. Nous verrons plus loin qu'elle est clairement établie dans les discours rituels convoquant les âmes.

L'âme du mort a donc une "maison ", mais sa fonction diverge selon les groupes considérés. Pour les Lacandons, la volonté des vivants est d'éloigner le défunt. Ainsi, après lui avoir construit un abri en forêt, on prend soin de son âme pendant quelques jours, tout en évitant son contact ou, pire, son retour parmi sa famille, jusqu'à ce qu'elle ait définitivement quitté l'espace des hommes. Le dessein des Yucatèques est tout autre. La maison du mort est aussi celle des vivants et, loin de chercher à en éloigner l'âme, on favorise son retour. Nous verrons bientôt quand et comment s'effectue le retour de l'âme. Signalons d'ores et déjà que la maison est l'espace privilégié de réunion des hommes et des morts. Nous avons mentionné plusieurs exemples ethnographiques prouvant que l'endroit où se trouvent les os (que nous considérons comme des reliques) correspond au lieu par lequel l'âme arrive sur terre et où elle reste pendant son séjour ici-bas. Cet endroit, que nous nommons "sa maison », les Yucatèques le font coïncider avec la maison familiale. Dans cette dernière se trouve également l'autel à partir duquel on appelle l'âme du défunt et où sont placées les offrandes. 
Toutefois, ce système apparaît vraiment cohérent que si l'on se réfère aux usages du passé. Ce que nous décrivons ici devait exister à l'arrivée des Espagnols et plusieurs changements sont intervenus à date plus ou moins récente selon les régions. Le plus significatif d'entre eux a été l'implantation de cimetières, lesquels ont rejeté les reliques hors de la maison, dissociant les espaces physiques des morts (la tombe) et des vivants (la maison). Une observation de Pacheco Cruz éclaire la situation passée : les Mayas du Quintana Roo «éprouvent une grande satisfaction à avoir leurs morts près d'eux et gardent ainsi l'impression de vivre en famille $»^{32}$.

L'unité de l'habitation familiale yucatèque concentre théoriquement non seulement tous les descendants du patriarche vivant, en ligne patrilinéaire, mais aussi tous ses ascendants non vivants. La maison devient ainsi une manifestation tangible de la descendance et de la lignée. De nos jours, l'expression la plus prégnante de cette affirmation de la lignée a lieu lors du hanal pixan, à partir du $1^{\text {er }}$ novembre, où les âmes des morts coexistent avec les vivants dans un espace commun, celui de la maison.

\section{LE RETOUR DES MORTS ET LE RITE DU HANAL PIXAN}

Il faut à présent se pencher sur les diverses interventions des âmes dans la vie des vivants et les cérémonies réalisées par ces derniers pour leurs morts. Nous nous attarderons sur le principal rituel effectué pour les âmes des défunts dans la péninsule du Yucatán, celui du hanal pixan, qui débute le jour de la fête des morts.

\subsection{Les rites familiaux et l'influence des morts}

Chez les Lacandons, l'influence des morts sur les vivants est limitée car elle se fait toujours par l'intermédiaire des dieux. C'est le rite de divination qui révèle aux vivants que les âmes de certains défunts se sont plaintes aux dieux des mauvais traitements subis durant leur vie, attirant la colère et le châtiment divins sur leurs proches. Ainsi l'histoire d'un vieil époux qui, délaissé par sa jeune compagne, meurt de tristesse. Après qu'il en a informé les dieux, ceux-ci font mourir tous les enfants que cette femme a avec son second mari (Boremanse, communication personnelle). Les Lacandons ne prennent guère soin des âmes des morts, n'entretenant pas de relation avec les défunts : ils ne leur rendent aucun culte particulier.

Pour les Yucatèques, les morts ne se manifestent généralement aux vivants que par l'intermédiaire du rêve ou de la maladie. Ils font part de leur mécontentement et manifestent la volonté d'être honorés, en particulier par des offrandes de nourriture (Redfield et Villa Rojas 1934). Pour les habitants de x-K'opch'en, avec qui nous avons eu l'occasion d'aborder ce sujet, les âmes des morts sont, 
comme des vents ( $i k^{\prime}$ ), invisibles. Le seul espace de rencontre possible est donc le rêve mais la communication est limitée. La maladie permet parfois d'entrer en contact avec elles. Ainsi l'histoire de cette grand-mère, très malade, qui entendit des âmes entrer chez elle, manifestant leur volonté qu'on fasse des offrandes en leur honneur (Archive sonore personnelle). Les morts yucatèques, s'ils ne sont pas honorés correctement, peuvent envoyer maladie ou infortune. Pour des troubles inexpliqués, on consulte donc un h-men (littéralement « faiseur », nom donné au chamane maya) qui, grâce à des instruments de divination (cristaux, cartes à jouer, grains de maïs, etc.), pourra préciser si le mal est dû à un parent décédé et indiquer quel rituel accomplir.

Des rites sont aussi réalisés régulièrement, sans forcément qu'ils répondent explicitement à un désir du mort. Villa Rojas (1978) témoigne d'une pratique des Mayas du Quintana Roo qu'il assimile lui-même à un culte des ancêtres : la ets'unah-gracia. C'est une prière réalisée assez fréquemment par un individu ou par la famille tout entière, parfois à l'église mais, le plus souvent, dans l'oratoire familial ou dans la maison. Ets'unah-gracia signifie littéralement « disposer ou offrir la grâce ou une offrande » et s'effectue lorsque les intéressés considèrent le moment approprié pour " un hommage à la croix familiale et domestique ou aux âmes de leurs ancêtres, spécialement, de leurs parents » ${ }^{33}$. Cette cérémonie peut être accomplie à n'importe quelle heure de la journée et, aussi, en l'absence d'un maestro cantor (représentant religieux chrétien qui récite d'habitude les prières). Ce dernier point renforce l'idée d'un culte privé, familial ou individuel, des ancêtres. Des offrandes de nourriture sont faites aux âmes, elles seront ensuite réparties entre la famille et les voisins les plus intimes. Le rezo (de l'espagnol " prière ») est aussi une cérémonie à caractère privé et accomplie comme la précédente dans la maison ou l'oratoire familial. Le rezo est récité pour commémorer la mort d'un membre de la famille. Il a lieu lorsque l'on suppose qu'une douleur ou une maladie a été causée par l'âme d'un ancêtre contrarié. De même, les offrandes sont partagées à la fin du rituel. Il existe un autre type de rezo, pour le repos de l'âme du mort, qui doit être fait à des dates déterminées et en présence d'un maestro cantor et des proches du défunt. Une autre cérémonie consiste à faire des offrandes de trois calebasses d'atole à l'âme du mort au moment du déjeuner. Les vieilles femmes que Villa Rojas a observées donnaient l'impression de converser avec leurs parents défunts (op. cit., p. 345 ; Redfield et Villa Rojas 1934).

\subsection{Le hanal pixan}

Si les morts peuvent se manifester tout au long de l'année, il est une période particulière, pour les Mayas-Yucatèques, pendant laquelle les âmes reviennent parmi les leurs : c'est le moment que l'on nomme hanal pixan, littéralement « la nourriture des âmes "). 


\subsubsection{Historique}

On peut s'interroger sur l'origine de ce rituel qui ne figure jamais explicitement dans les sources coloniales (Tozzer 1941 ; Roys 1943 ; Farriss 1984). Notons qu'il s'agit d'une cérémonie d'ordre familial et privé. Or les chroniqueurs se sont surtout intéressés aux cultes publics et à ceux des classes sociales élevées - c'étaient celles avec lesquelles ils étaient le plus en contact. De plus, la christianisation a sans doute largement influencé la forme du rituel et, surtout, la date à laquelle il est accompli. En effet, il est réalisé à la Toussaint. Les premiers ethnologues, tels Gann (1918) ou Thompson (1930), qui rapportent cette pratique, expliquent que chez les Mayas-Mopan « on pense vaguement que les morts reviennent dans ce monde pour la Toussaint $»{ }^{34}$.

Si l'on considère que le jour des morts maya est une fête syncrétique, il est alors nécessaire de rappeler les origines européennes de cette célébration afin de mieux comprendre les éventuelles adaptations réalisées lors de l'évangélisation. Il semble que cette fête trouve sa source dans des rites celtes, bien qu'il existât déjà en Égypte un jour des morts, sous l'égide d'Osiris, lors du solstice d'hiver. De la nourriture était préparée pour les âmes des morts ; des lampes à huile étaient disposées sur les façades afin d'éviter qu'elles ne s'égarent. De même, en Grèce et à Rome, de la nourriture était offerte aux âmes des défunts vers le mois de février. Les premières traces d'un rituel pour les morts chez les chrétiens concernent les martyrs sur la tombe desquels on allait manger et déposer des offrandes. Cette pratique fut interdite au VIII $^{\mathrm{e}}$ siècle par Grégoire III qui décida de consacrer le $1^{\text {er }}$ novembre à la mémoire des martyrs. C'est au XI ${ }^{\mathrm{e}}$ siècle que le jour du 2 novembre fut consacré aux morts non canonisés.

Dans la fête européenne, la présence de nourriture est aussi une constante. Ainsi, dans l'Italie du XIv ${ }^{\mathrm{e}}$ siècle, les habitants dressaient une table de nourriture et laissaient la maison aux esprits pendant qu'ils partaient pour la journée à l'église. En réalité, ce sont les mendiants et les vagabonds qui en profitaient. Au $\mathrm{xv}^{\mathrm{e}}$ siècle, l'Église tenta d'interdire l'usage de la nourriture, considéré comme une marque de paganisme, mais elle ne parvint seulement qu'à le limiter (Foster 1962). Jusque récemment en Espagne, le pan de los muertoslánimas était encore offert aux mendiants le $1^{\mathrm{er}}$ novembre, de même que des fleurs et de la nourriture étaient placées sur les tombes (Linton et Linton 1950). Cela pourrait expliquer la tolérance des religieux espagnols, lors de la Conquête, vis-à-vis des pratiques mayas où la nourriture était présente. Les Indiens auraient donc pu greffer certains de leurs usages sur le rite espagnol.

\subsubsection{Le rituel}

Pour présenter le rituel, nous nous appuierons sur les descriptions fort précises de Redfield et Villa Rojas (1934 et 1978) qui l'ont observé au Yucatán et au 
Quintana Roo. Chez les Itza', le rituel des trois crânes, décrit par Reina (1962), s'insère dans le hanal pixan mais ne concerne que les quelques familles de la communauté qui ont choisi d'accueillir les crânes une partie de la nuit du $1^{\text {er }}$ novembre.

Les préparatifs commencent le 30 octobre, la nourriture est cuisinée et les fleurs sont cueillies. À minuit, on dispose sur les tables des fleurs, les mêmes que celles ornant les portes des maisons, comme une invitation aux âmes à entrer. Sur la table, sont arrangés du chocolat, du pain et quelques bougies, toujours en nombre pair. Les voisins qui sont conviés à cette cérémonie deviendront, plus tard, eux aussi amphitryons. Une calebasse d'eau, dite $x$-p'o $k^{\prime} a b$, servant à se laver les mains, est également placée comme offrande pour les morts. Une calebasse de boisson chocolatée, un morceau de pain et une bougie ont été posés sur le pas de la porte, pour les âmes qui n'ont plus de famille. Les âmes qui se présentent en premier sont celles des enfants. Elles arrivent très tôt le 31 octobre, jour considéré comme celui des enfants ${ }^{35}$. Le premier repas qu'elles prendront sera leur déjeuner. Au Quintana Roo, cette cérémonie se déroule à l'église et dans les oratoires familiaux alors qu'au Yucatán, la maison est l'unique lieu de célébration. C'est le maestro cantor qui se rend dans chaque habitation et y récite les prières adéquates. Ensuite, la nourriture placée sur les tables est consommée par les personnes présentes. Plus tard dans la journée, le rituel sera réitéré, les âmes dîneront d'abord, puis ce sera au tour des vivants. La nuit venue, on allumera des bougies sur chaque tombe, afin que les âmes des enfants (les angeles) puissent retrouver leur chemin. Thompson signale que « ce jour-là des bougies sont allumées à l'aube et au crépuscule, placées aux quatre coins de chaque maison et un petit peu de copal est brûlé » ${ }^{36}$.

Le jour suivant est celui de l'arrivée des âmes des adultes. Elles sont reçues dans les mêmes conditions que celles des enfants. Des prières spécifiques leur sont destinées. Les tombes sont balayées et garnies de fleurs. L'après-midi, l'hommage se fait à la maison où un autel est improvisé et sur lequel on place, selon les termes de Villa Rojas (1978, p. 432), la cruz « fiadora » de la familia [la croix garante de la famille]. Le soir, il n'est pas nécessaire d'allumer des bougies sur les tombes des adultes car leurs âmes sont « assez grandes pour retrouver facilement leur chemin » (ibid.). Toutefois, nous avons pu observer qu'actuellement, au Quintana Roo, on place des bougies à chaque cérémonie sur les murs entourant le sòolar afin que les âmes des morts, enfants comme adultes, trouvent leur chemin. Au Yucatán, les gens se rendent au cimetière le 2 novembre, emportant avec eux les restes des bougies utilisées pour les rituels domestiques et qu'ils allumeront sur les tombes fleuries. Pendant la semaine où les âmes errent, on craint pour la santé des enfants. C'est pourquoi on noue un bracelet de ficelle autour des poignets des plus jeunes afin d'éviter qu'ils ne soient emportés par celles-ci. À la fin de cette huitaine (octava), elles sont renvoyées avec les mêmes égards que ceux dispensés lors de leur accueil. Le 7 novembre, ce sont les âmes des enfants qui partent et, le 
lendemain, celles des adultes. Au Quintana Roo, les célébrations durent jusqu'au 24 décembre et deux offrandes sont nécessaires pour chaque mort : l'une composée de tamales bouillis, l'autre de tamales cuits sous la terre (Vapnarsky, communication personnelle).

À $x$-K'opch'en, les choses se passent d'une manière quelque peu différente. D'abord, les âmes des enfants - en réalité il s'agit des âmes des jeunes non mariés - arrivent le $1^{\text {er }}$ novembre ; puis, le jour suivant, celles des adultes. Mais toutes se rassemblent dans un premier temps à l'église où est réalisé un máatan en leur honneur. La nourriture est pour toutes les âmes (tuláakal le pixano'obo') qui ne mangeront que « l'odeur » des aliments. En fait, il semble que l'offrande soit destinée à San Juan Bautista, le patron du village, qui prendra soin de répartir lui-même la nourriture entre elles. La Mort elle-même est aussi invitée, c'est en quelque sorte la gardienne des morts (uRey-il) lors de leur séjour sur terre. Mais, étant donné sa voracité, on ne la cite qu'en dernier lieu et on lui donne des choses dures à manger (os de poulet, œuf dur avec la coquille, etc.) afin de prolonger son repas et de laisser le temps aux âmes de se repaître. Ce sont les âmes présentes sur l'ejido (c'est-à-dire sur les terres du village) qui sont conviées, y compris celles des villages abandonnés (San Pedro, Chan X-K'opch'en et Sahkabch'en), toutes étant sous la protection de San Juan. La nourriture sera ensuite entièrement distribuée à l'assistance. Dans un deuxième temps, chaque famille fera un máatan dans la maison familiale, conviant ses propres morts : parents, frères, enfants. Les morts sont appelés individuellement, par leur nom, et une prière spéciale est récitée pour chacun d'eux. S'il y a une dizaine d'âmes, les prières peuvent durer des heures. Ces cérémonies familiales sont réalisées jusqu'à la date du 24 décembre. Pendant cette période, les âmes des morts errent sur terre, dans le village. Selon nos informateurs, lorsqu'elles reviennent sur terre, elles sont affamées et mangent toute la nourriture qui est présente (c'est-à-dire le pixan des aliments). Selon certains, c'est comme si les âmes mangeaient en rêve ; la nourriture est toujours présente dans la réalité des hommes. Cependant, lors des cérémonies familiales, les offrandes sont destinées en priorité aux âmes que l'on nomme et ce n'est que lorsque celles-ci sont repues que les autres peuvent à leur tour manger, reproduisant ainsi ce qui se passe pour les hommes quand, dans les máatan, on sert en priorité les nohoch máako'obo' (les hommes les plus importants ou ceux qui font l'office), puis les autres personnes.

Nous constatons que, contrairement aux morts catholiques, les âmes ont, chez les Yucatèques, une grande influence sur les vivants ; il est impératif de leur rendre hommage régulièrement. C'est le but notamment du rezo qui, bien qu'il soit nommé sous un terme d'origine espagnole, est loin de ressembler à la prière catholique pour le repos d'un mort. Il est destiné, chez les Yucatèques, à calmer le mécontentement d'un défunt afin qu'il cesse de perturber les vivants. Le ets'unahgracia, composé d'un terme espagnol et maya, est clairement désigné par Villa Rojas (1978) comme un hommage aux ancêtres. Quant au hanal pixan, malgré ses 
similitudes avec le jour des morts catholique, de nombreuses pratiques et conceptions diffèrent : on retiendra le fait que les âmes reviennent errer parmi leurs descendants vivants et manger avec eux pendant une période allant de neuf jours à deux mois, puis la séparation qui est faite entre les âmes des enfants et celles des adultes, enfin, le lieu du rituel qui est presque uniquement la maison et rarement l'église. Tout cela suggère qu'un rituel d'un autre ordre, un culte des ancêtres, pourrait être sous-jacent au jour des morts.

\section{Discussion}

Reprenons à présent, point par point, les critères évoqués en introduction à propos du culte des ancêtres tel qu'il est pratiqué dans les sociétés asiatiques ou africaines, et confrontons-les aux pratiques et croyances des Mayas des Basses Terres afin d'établir s'il est légitime de parler de culte des ancêtres. Dans un premier temps nous nous intéresserons uniquement aux Mayas-Yucatèques ; nous traiterons du cas des Lacandons dans une partie distincte.

\subsection{La mort ne fait pas l'ancêtre}

La mort ne suffit pas à conférer le statut d'ancêtre et, comme le souligne Hertz dès 1907, des rites spécifiques, en plus de l'inhumation, sont généralement nécessaires. Ils marquent à la fois un changement d'état tout en réaffirmant, du même coup, la cohésion interne du groupe. C'est le cas, par exemple, en Chine du sud où les restes du mort sont transférés dans une nouvelle sépulture, indiquant la transition du statut de mort à celui d'ancêtre (Freedman 1970).

Peut-on parler de doubles obsèques chez les Mayas-Yucatèques ? Un exemple, cité plus haut, pourrait être interprété comme tel. Il s'agit du rituel pratiqué au Yucatán trois ans après l'inhumation qui consiste à déterrer, lors du hanal pixan, les ossements du défunt pour les placer dans un reliquaire ailleurs dans le cimetière. Rappelons qu'on ne retrouve pas cette cérémonie au Quintana Roo. En revanche, ce qui est commun à tous les Yucatèques, ce sont les rites réalisés en l'honneur du mort pendant une année. Après la mort, on accomplit une série de rites qui s'achève à l'anniversaire du décès (kàabo de 'àanyo). C'est uniquement à la fin de cette période, ainsi qu'on nous l'a expliqué à x-K'opch'en, que l'âme peut redescendre sur terre et participer, comme les autres morts, aux cérémonies du hanal pixan. Le rite du $1^{\mathrm{er}}$ novembre correspond donc à la fin d'un processus pour l'âme jeune mais également au commencement d'un cycle où, chaque année, elle sera honorée par sa famille. Cette transformation du statut de l'âme peut être interprétée, selon nous, comme un processus d'ancestralisation. 


\subsection{Un nom attaché à une relique}

Pour Fortes (1965), l'ancêtre est, contrairement au mort, une entité identifiable et individualisée. Divers éléments sont nécessaires pour cette spécification du mort : le nom, c'est le cas en Afrique (Fortes 1965), et les reliques, fondamentales dans les cultes asiatiques (Freedman 1970).

Chez les Yucatèques, le corps est fondamental dans la relation que les vivants entretiennent avec l'âme de leurs parents, servant de médiateur entre le groupe des hommes et celui des ancêtres. Pour le défunt, c'est le point de passage entre l'univers des morts et celui des vivants. À l'époque postclassique, certaines reliques sont très élaborées, sous forme d'urnes ou de crânes décorés, dont la coutume itza pourrait être une réminiscence. Mais, dans la majorité des cas, les ossements situés dans l'unité d'habitation familiale remplissent le rôle de reliques, la maison celui de reliquaire. De cette façon, la famille identifie bien le mort, du moins jusqu'à la création des cimetières. La dissociation physique actuelle de la relique et de la maison n'a pas malgré tout fondamentalement altéré la relation entre homme et ancêtre car à la relique est également attaché un nom, dont le rôle est primordial dans les rituels. C'est par l'intermédiaire de ce nom que l'âme d'un ancêtre est convoquée et qu'on établit une hiérarchie entre les âmes des défunts. Le nom du défunt appartient uniquement à la mémoire familiale, il n'est d'ailleurs pas indiqué sur la croix de la tombe au cimetière.

Notons enfin l'importance de l'autel familial situé dans la maison, support essentiel du rituel. Goody (1962, p. 381) souligne d'ailleurs le lien entre la figure humaine de l'ancêtre et l'autel individuel. Chez les Yucatèques, ce dernier remplit désormais, après sa séparation physique d'avec les reliques ancestrales, un rôle équivalent à celui des tablettes chinoises. En contexte rituel, c'est en effet depuis l'autel que l'on convoque nominalement les ancêtres et c'est là qu'on leur dépose les offrandes.

\subsection{Le culte des ancêtres, une institution filiale et familiale}

Le culte des ancêtres est généralement enraciné dans les institutions de la famille et dans la parenté. Il a souvent pour objectif de spécifier un groupe d'individus qui se réclame d'un ancêtre particulier et se distingue ainsi de ses collatéraux (Fortes 1965; Goody 1962). La filiation est primordiale, car un ancêtre n'est défini que par rapport à une lignée (Fortes 1965). Chez les Yucatèques, nous pensons que la spécification du groupe et l'ancrage de l'ancêtre dans une lignée familiale peuvent être perçus à travers la commensalité, d'une part, et la maison, de l'autre.

Le partage de la table et de la nourriture est un acte social fort, comme le remarque Jordan (1972) à Taiwan où «les limites sociales de la famille sont rituellement exprimées en termes de commensalité ${ }^{37}$. De même, en Chine, on 
ne mange qu'avec les gens de la famille, des amis proches ou des invités de statut social identique. Les offrandes aux entités surnaturelles respectent les mêmes principes (Wolf 1974). Nous pensons, à l'instar de Wolf, que les MayasYucatèques appliquent avec les morts des règles de commensalité identiques à celles qui prévalent chez les vivants. C'est ainsi que les offrandes des rituels effectués pour les morts sont uniquement distribuées aux membres de la famille et aux amis proches. Toutes les âmes ne sont pas non plus conviées à les consommer. Seules celles qui font partie de la famille et pour qui on récite une prière nominative - autrement dit les ancêtres familiaux - en sont les destinataires.

La maison, nous l'avons montré, constitue un ancrage territorial à la lignée, une manifestation physique de la cognation. Elle fut l'endroit où étaient entreposées les reliques et reste le lieu normal du départ de l'âme mais surtout celui de son retour. Lors de certains rituels, notamment le hanal pixan, elle constitue un espace privilégié où se rencontrent les ancêtres et leurs descendants. Cette contiguïté permet sans doute une réaffirmation du groupe et de son identité, mais il nous est difficile de dire dans quelle mesure.

Certains auteurs, Gillespie notamment, insistent sur la relation entre lignage et maison, proposant même de substituer le second terme au premier dans les analyses archéologiques concernant les Mayas classiques. Ils considèrent les unités résidentielles comme reflétant le lignage cognatique dont les principes d'organisation de la descendance auraient pour fondement, entre autres, le culte des ancêtres. L'intention de leur réflexion est de reconsidérer les lignages mayas classiques sur le modèle lévi-straussien de la maison (pour une discussion sur ce sujet, voir Gillespie 2000a ; 2000b et Chance 2000). Lévi-Strauss (1984, p. 190 ; 1979) suggère, pour expliquer certains systèmes de parenté, d'utiliser la notion médiévale de maison, c'est-à-dire de personne morale, détentrice d'un domaine qui se perpétue par transmission de son nom et de ses biens s'inscrivant dans une lignée réelle ou fictive et s'exprimant dans le langage de la parenté et/ou de l'alliance. Pouvons-nous appliquer un tel modèle dans le cas des MayasYucatèques? Actuellement, cela paraît difficile car la transmission des biens et des noms est intégrée dans le système légal mexicain (testament, lois foncières, etc.) et le culte des ancêtres ne peut plus constituer une justification suffisante. En revanche, il semble que les nobles mayas préhispaniques - les Cocom par exemple - pratiquaient un culte des ancêtres qui rendait légitime leur statut social et politique ${ }^{38}$. Gillespie $(2000 \mathrm{~b}, \mathrm{p}$. 475) souligne à cet égard que ces « actions dirigées vers les corps et les esprits des ancêtres étaient utilisées pour créer des différences politiques et sociales entre les nobles et les gens du commun ainsi qu'entre les divers groupes de nobles ${ }^{39}$. Mais, là encore, il est délicat d'inférer plus avant à partir de données limitées que nous avons concernant cette époque. 


\subsection{Les morts lacandons}

Nous constatons, à travers cette étude comparative, que les Lacandons ont face à leurs morts une attitude divergente par rapport aux autres groupes yucatèques. Si certaines croyances sont communes, pour les Lacandons la fin de la période liminale met un terme à la relation entre vivants et morts. Jusqu'à cette date, l'âme du défunt est en étroite relation avec son corps qui permet de la nourrir ; les règles de commensalité exposées plus haut l'intègre encore dans le groupe familial, même si, déjà, la tombe érigée dans la forêt et la volonté de tenir le mort à l'écart présagent la rupture prochaine. Lorsque celle-ci a lieu, l'âme rejoint l'espace des morts, un univers totalement dissocié de celui des vivants. Autrefois, comme le relatent les mythes (Boremanse 1986), des passages reliaient les deux mondes, c'est par là que des hommes se sont rendus au royaume des morts et c'est grâce à leurs témoignages que l'on connaît son existence. Puis ces passages ont été fermés. Les morts vivent en communauté, chez Mensabäk (dans l'inframonde) ou chez les Wayantekob (au ciel), et jamais ils ne sont amenés à revenir parmi les vivants. Cela expliquerait l'absence de rituel tel que le hanal pixan visant à accueillir régulièrement les âmes des morts.

\section{CONCLUSION}

À partir de critères caractérisant le culte des ancêtres dans diverses sociétés, on a pu suggérer son existence chez les Mayas-Yucatèques. L'analyse comparative esquissée a néanmoins permis de mettre en évidence que tous les groupes mayas des Basses Terres ne le partagent probablement pas. Tel est le cas des Lacandons qui n'accomplissent aucun rituel particulier pour les morts après la fin de la période liminale. Chez eux, les dieux sont les intermédiaires pour entrer en relation avec les défunts, lesquels ne reviendront jamais dans le monde des hommes. Cette divergence entre les morts lacandons et les ancêtres yucatèques surprend quelque peu eu égard à leur proximité culturelle et surtout linguistique.

Si l'on peut faire remonter l'existence d'un culte des ancêtres à la période préhispanique, il apparaît qu'il revêtait à cette époque une forme et des desseins bien différents du rite actuel. Les témoignages coloniaux démontrent que le culte des ancêtres permettait, notamment aux personnes de rang élevé, de préserver leur statut social et politique d'une génération à l'autre ; ce modèle préhispanique a subi de nombreuses évolutions. Les premières sont dues évidemment à la christianisation, à l'arrivée des Espagnols ; les Mayas-Yucatèques ajustent alors leurs pratiques et leurs croyances dans les espaces que leur laisse le culte hispanique, c'est-à-dire le jour des morts et les prières. Plus tard, les lois mexicaines et la modernisation influenceront également les pratiques funéraires, en particulier avec la généralisation de l'inhumation au cimetière. L'importance donnée 
aujourd'hui à ce culte va néanmoins bien au-delà de la simple commémoration des morts et, sous jacente aux pratiques syncrétiques, subsiste une pensée maya qui ne voit dans la mort qu'un changement d'état. Les ancêtres non seulement perdurent dans la mémoire des vivants mais coexistent régulièrement avec eux. *

* Manuscrit reçu en septembre 2002, accepté pour publication en février 2003.

\section{NOTES}

Remerciements : Je tiens à exprimer ma gratitude à Valentina Vapnarsky pour sa patience et ses commentaires, ainsi qu'à remercier Alain Breton, Pierre Becquelin et Jacques Galinier pour leurs remarques. Mes remerciements vont aussi à Didier Boremanse et Mario Humberto Ruz pour leur précieuse aide, à María Alejandra Pérez, à Sophie Ménoret et à toute l'équipe du laboratoire de I'EREA. Ma reconnaissance va aussi à Délio Chan Chi pour son aide et son soutien sur le terrain.

Avertissement : Dans le texte, toutes les citations en langue étrangère ont été traduites par l'auteur (O.L.G.). Nous en donnons la référence exacte et la version originale en note.

1. "An ancestral "spirit» is not thought of as a kind of nebulous being or personified mystical presence but primarily as a name attached to a relic [...] standing for ritual validation of lineage ancestry and for mystical intervention in human affairs. 》 (Fortes 1987, p. 72)

2. "If ancestor worship is subsumed under the worship of the dead then its meaning must be sought in customary beliefs and pratices concerning death, the soul, ghost, spirits and the after-life. " (ibid.)

3. Notons qu'il existe aussi de nombreux dictionnaires coloniaux de la langue yucatèque (voir notamment Álvarez 1984). Toutefois leur intérêt reste assez limité pour notre étude car ils nous renseignent bien évidemment sur le vocabulaire mais n'apportent que peu d'informations au niveau ethnologique.

4. Les travaux ethnologiques plus récents n'abordent pas le thème qui nous occupe ici (voir, par exemple, Sullivan 1989 et Burns 1983).

5. Sur les cartes et les panneaux mexicains, le nom du village s'écrit Kopchen mais nous préférons transcrire le nom tel qu'il est prononcé dans la région par les Mayas eux-mêmes.

6. On retrouve ce nom de Yum Kimil pour désigner la mort dans des contes mayas actuels ou dans des récits de tradition orale.

7. Les encensoirs chez les Lacandons permettent d'adresser des prières aux dieux.

8. Sabal spp.

9. Cette croyance se retrouve dans le Popol Vuh des K'iche' des Hautes Terres du Guatemala.

10. Le balche' est une boisson cérémonielle. Il s'agit d'un mélange d'eau et de jus de canne à sucre ou de miel dans lequel on laisse tremper des bandes d'écorce de l'arbre balche' pendant vingt-quatre heures.

11. Hach Ak Yum est le dieu créateur des Lacandons septentrionaux, c'est aussi leur principale divinité.

12. Kisin est le seigneur de l'inframonde (Metnal) chez les Lacandons. Pour les Yucatèques actuels, c'est le diable ; à $x-K$ 'opch'en, il répond au stéréotype de l'être entièrement rouge avec une queue et des cornes.

13. De la famille des palmes (PalmaelArecaceae).

14. Les enfants morts dans leur jeune âge sont nommés angeles ("anges») auxquels ils sont comparés.

15. "A los señores y gente de mucha valia quemaban los cuerpos y ponían las cenizas en vasijas grandes, y edificaban templos sobre ellas [...]. Se halló que echaban las cenizas en estatuas huecas, hechas de barro, cuando eran muy señores. La demás gente principal hacia a sus padres estatuas de madera a las cuales dejaban hueco el colodrillo, y quemaban algma parte de su cuerpo y echaban alli las cenizas y 
tapábanlo ; y después desollaban al difundo el cuero del colodrillo y pegabánselo alli, enterrando los residuos como tenian de costumbre ; guardaban estas estatuas con mucha reverencia entre sus idolos. „ (Landa 1985, p. 101)

16. "A los antiguos señores Cocom, habian cortado las cabezas cuando murieron, y cocidas las limpiaron de la carne y después aserraron la mitad de la coronilla para atrás, dejando lo de adelante con las quijadas y dientes. A estas medias calaveras suplieron lo que de carne les faltaba con cierto betín y les dieron la perfeccion muy al proprio de cuyas eran, y las tenian con las estatuas de las cenizas, con sus idolos, en muy gran reverencia y acatamiento." (ibid.)

17. Tozzer souligne en note que des statues de bois et des crânes coupés, peints et travaillés pour être ressemblants, ont été retrouvés dans le puits des sacrifices de Chichén Itzá (Tozzer 1941, p. 131, notes 612-613 ; voir aussi Coggins 1992). McAnany (1995) voit, quant à elle, dans le codex de Madrid, des représentations du processus de fabrication de ces reliques (pp. 96d et $97 \mathrm{~b}$ du codex de Madrid [Tro-cortesianus]).

18. "They are more important than the saints because the saints are made of wood. The skulls have been human beings and have been alive. They are real bones just as ours are. " (Reina 1962, p. 32)

19. "They put them in a shroud, filling their mouths with ground of maize, which is their food and drink which they called koyem » (Tozzer 1941, p. 130). Cet auteur précise en note 605 qu'il s'agit de pozole.

20. "Until recently the personal possessions of the dead man and food were buried with him, but this custom is gradually dying out. " (Thompson 1930, p. 82)

21. "It is still customary to throw away a little food at the end of the third after the death. To do this the relative or friend of the deceased proceeds a short way into the wood and throws the offering away. " (Ibid., pp. 81-82)

22. Sukunkyum est le seigneur du bas monde dans la mythologie des Lacandons méridionaux.

23. Mensabäk est, pour les Lacandons septentrionaux, le gardien des âmes des morts. Il vit dans une grotte près du lac qui porte son nom.

24. "Food played a major role in all the colonial Maya's solemn observances. Communal banquets accompanied all the fiestas, the wakes and other observances for the dead, the rain-making Cha-Chaac ceremonies and more apparently secular occasions such as weddings and roof raisings. Commensality clearly serves social purposes. Shared meals establish the boundaries of a group, express its collective identity, and sustain its internal cohesion. " (Farriss 1984, p. 321)

25. "Enterrábanlos dentro de sus casas o a las espaldas de ellas. " (Landa 1985 ; voir aussi Tozzer 1941 , p. 130 , note 607 )

26. "Thirty or forty years ago people were often buried beneath the floor of their houses. " (Thompson 1930, p. 82)

27. " En otras comunidades utilizan hasta las mismas iglesias » (Pacheco Cruz 1934 ; 1947). Cela dit, il ne s'agit en rien d'une pratique exceptionnelle. Voir, par exemple, ce témoignage sur la Colombie du $\mathrm{XIX}^{\mathrm{e}}$ siècle : « peu de villes ont encore des cimetières publics et, jusque dans les années 1820 au moins [...], on ensevelit les corps sous les pavés des églises, ainsi qu'on le faisait en France à l'époque médiévale, [on] voit aussi des tombes sur le bord des champs près de Serinsa dans le Socorro »(Le Guen 1999,p. 93).

28. Voir, notamment dans Boremanse (1986, p. 80), le mythe dans lequel le chasseur de taupe descend dans l'inframonde et éloigne Kisin (le seigneur des lieux) avec du piment grillé.

29. "The most important of which are the warabal ja (sleeping house) of the ancestors. " (Carmack 1981, p. 161)

30. Bien que ne rentrant pas dans la période chronologique que nous avons choisi de considérer, on ne peut pas ne pas évoquer les temples-pyramides de l'époque classique comme ceux de Palenque ou Tikal contenant des tombes de rois.

31. " Por su investidura lo consideraron digno a perpetuarse en aquel lugar i no en la calle. " (Pacheco Cruz 1947, p. 28)

32. "Experimentaban gran satisfacción en tener a sus deudos cerca y conservaban la impressión de vivir en familia. " (ibid., p. 27) 
33. "Un homenaje a la cruz familiar o a las almas de sus ancestros, especialmente, de los padres." (Villa Rojas 1978, pp. 343-344)

34. "The dead are vaguely believed to return to this world on All Souls' Day. " (Thompson 1930, p. 82)

35. Chez les Aztèques, il existait aussi un jour des enfants, le miccailhuitontli, ou « fête des petits morts », qui introduisait la " fête des grands morts » (Binant 1982).

36. "On that day candles are lit at dawn and at dusk, and are placed in the four corners of each house, and a little copal is burnt. " (Thompson 1930, p. 82)

37. "The social boundaries of the family are ritually set forth in terms of commensality. " (Jordan 1972, p. 89)

38. Le pouvoir politique, à l'époque postelassique, était entre les mains de grandes familles (dont les Cocoms et les Xiu) et était transmis de manière héréditaire.

39. "Actions directed toward ancestors' bodies and spirits were used to create social and political differences between nobles and commoners and among different noble groups. "(Gillespie 2000b, p. 475)

\section{BIBLIOGRAPHIE}

AHERN Emily M.

1973 The Cult of the Dead in a Chinese Village, Stanford University Press, Stanford.

Álvarez Cristina

1984 Diccionario etnolingüístico del idioma maya yucateco colonial, UNAM, Mexico, 3 vols.

BastarRachea Juan R.

1992 Diccionario básico español-maya-español, Ediciones Maldonado, Mérida.

Binant Pascale

1982 La Toussaint, jour des morts : l'objet dans la fête mexicaine, mémoire de maîtrise d'ethnologie, Paris X-Nanterre.

BOREMANSE Didier

1986 Contes et mythologie des Indiens lacandons, L'Harmattan, Paris, coll. "Connaissance des Hommes ».

1998 Hach Winik, The Lacandon Maya of Chiapas, Southern Mexico, Institute for Mesoamerican Studies, State University of New York, Albany, NY. coll. « Monograph 11 ».

BRETON Alain

1991

« Un besoin infini d'ancêtres », in A. Breton et J. Arnauld, eds, Mayas. La passion des ancêtres, le désir de durer, Éditions Autrement, Paris, pp. 150158.

Bricker Victoria R., Eleuterio Po'ot YaH et Ofelia Dzul de Po'ot

1998 A Dictionary of the Maya Language as Spoken in Hocabá, Yucatán, University of Utah Press, Salt Lake City.

BRUCE Robert D.

1968

Gramática del lacandón, Instituto Nacional de Antropología e Historia, Mexico. 
Bruce Robert D., Carlos Robles Uribe et María Elena Ramos C.

1971 Los lacandones. 2. Cosmovisión maya, Instituto Nacional de Antropología e Historia, Mexico.

BuRNs Allan F.

1983 An Epoch of Miracles: Oral Literature of the Yucatec Maya, University of Texas Press, Austin.

1993 "Voz y poesía en la filosofia maya-yucateca de la muerte », in J. Ponce de León et F. Ligorred Perramon, eds, Perspectivas antropológicas en el mundo maya, Sociedad Española de Estudios Mayas, Instituto de Cooperación Iberoamericana, Madrid, pp. 479-486, coll. « Publicaciones » 2.

CARMACK Robert M.

1981 The Quiché Mayas of Utatlán, University of Oklahoma Press, Norman.

Chance John K.

2000 "The noble house in colonial Puebla, Mexico : Descent, Inheritance, and Nahua Tradition », American Anthropologist, 102 (3), pp. 485-502.

Cogains Clemency C., ed.

1992 Artifacts from the Cenote of Sacrifice, Chichén Itzá, Yucatán, Harvard University Press, Cambridge, Mass., coll. "Memoirs of the Peabody Museum of Archaeology and Ethnology » 10 (3).

De Vos Jan

1988 La Paz de Dios y del Rey, la conquista de la selva lacandona (1525-1821), CIESAS, Mexico.

DURKHEIM Émile

1912 Les formes élémentaires de la vie religieuse, Alcan, Paris.

FARRISs Nancy M.

1984 Maya Society Under Colonial Rule. The Collective Enterprise of Survival, Princeton University Press, Princeton.

Figuerola Hélios

2000 "El cuerpo y sus entes en Cancuc, Chiapas », Trace, 38, pp. 13-24.

FORTES Meyer

1965 "Some Reflections on Ancestor Worship in Africa », in African Systems of Thought, study presented and discussed at the Third International African Seminar in Salsbury, December 1960, International African Institute, Oxford University Press, Londres, New York and Toronto.

1987 Religion, morality and the person : essays on Tallensi religion, Cambridge University Press, Cambridge and New York.

Foster George M.

1962 Cultura y conquista, Universidad Veracruzana/Talleres Gráficos de la Nación, Xalapa.

FreEdMan Maurice

1966 Chinese Lineage and Society : Fukien and Kwangtung, The Athlone Press, Londres. 
1970

Family and Kinship in Chinese Society, Stanford University Press, Stanford.

GanN Thomas W. F.

1918 The Maya Indians of Southern Yucatan and Northern British Honduras, Smithsonian Institution, Bureau of American Ethnology, Bull. 64, Washington.

GiLLESPIE Susan D.

2000a "Beyond kinship : An introduction », in R. A. Joyce et S. D. Gillespie, eds, Beyond Kinship : Social and Material Reproduction in House Society, Pennsylvania University Press, Philadelphia.

2000b "Rethinking Ancient Maya Social Organisation : Replacing "Lineage" with "House" ", American Anthropologist, 102 (3), pp. 467-484

Goody Jack

1962

Death, Property and the Ancestors. A Study of the Mortuary Customs of the Lodagaa of West Africa, Stanford University Press, Stanford.

Hertz Robert

$1970 \quad$ Contribution à une étude sur la représentation collective de la mort, Presses Universitaires de France, Paris [1907].

Jordan David K.

1972 Gods, Ghosts and Ancestors, the Folk Religion in a Taiwanese Village, University of California Press, Berkeley, Los Angeles and Londres.

KRICKEBERG Walter

1962 Les religions amérindiennes, Payot, Paris.

LANDA Diego de

1985 Relación de las cosas de Yucatán, édition de Miguel Rivera, Madrid, coll. « Historia » 16, [1566].

2000 Yucatan, Before and After the Conquest, traduit et annoté par William Gates, Dover Publications Inc., New York.

Le Guen Olivier

1999 La Colombie de la première moitié du xIX siècle vue par des voyageurs français, mémoire de maîtrise, Université de Nantes, Nantes.

LÉvi-STrauss Claude

$1979 \quad$ La voie des masques, Plon, Paris.

1984 Paroles données, Plon, Paris.

Linton Ralph et Adelin Linton

$1950 \quad$ Halloween Through Twenty Centuries, H. Schumann, New York.

McAnany Patricia A.

1995 Living With the Ancestors. Kinship and Kingship in Ancient Maya Society, University of Texas Press, Austin.

PACHeCo Cruz Santiago

1934 Estudios etnográficos de los mayas del ex-territorio Quintana Roo, [s. e.], Mérida.

1947 Usos, costumbres, religión i supersticiones de los Mayas, [s. e.], Mérida. 
Pitarch Pedro

1996 Ch'ulel : une etnografia de las almas tzeltales, Fondo de cultura económica, Mexico.

\section{REDFIELD Robert et Alfonso VILLA Rojas}

1934 Chan Kom, a Maya Village, Carnegie Institution, Washington, coll. « Publication » 448.

ReINA Ruben E.

1962 «The ritual of the skull », Expedition, 4 (4), pp. 27-35.

Roys Ralph L.

1943

The Indian Background of Colonial Yucatan, Carnegie Institution, Washington, coll. « Publication » 548.

Ruz Mario Humberto

2003 "Pasajes de muerte, paisajes de eternidad », in A. Breton, A. Monod Becquelin et M. H. Ruz, eds, Espacios mayas: usos, representaciones, creencias, Centro de Estudios Mayas, UNAM/CEMCA, Mexico, pp. 619657.

SMITH Robert J.

1974 Ancestor Worship in Contemporary Japan, Stanford University Press, Stanford.

Sullivan Paul R.

1989 Unfinished Conversations: Mayas and Foreigners Between Two Wars, Alfred A. Knopf, New York.

THOMPSON J. Eric S.

1930

Ethnology of the Mayas of Southern and Central British Honduras, Field Museum of Natural History, Chicago, coll. « Anthroplogical Series » 17.

1977 «A proposal for constituting a Maya Subgroup, Cultural and Linguistic in the Petén and Adjacent Regions ", in G. D. Jones, ed., Anthropology and History in Yucatan, The University of Texas Press, Austin et Londres, pp. 3-42.

Tozzer Alfred

1907 A Comparative Study of the Mayas and the Lacandones, Archaeological Institute of America, by MacMillan Company, New York.

1941 Landa's Relacion de las Cosas de Yucatan : A Translation, The Museum, Cambridge, Mass., coll. « Harvard University. Peabody Museum of American Archaeology and Ethnology. Papers » 18.

TYLOR Edward B.

1920 Primitive Culture, Putnam, New York [1871, Murray, Londres].

VAPNARSKY Valentina

2000 «De dialogues en prières, la procession des mots », in A. Monod Becquelin et P. Erikson, eds, Les rituels du dialogue. Promenades ethnolinguistiques en terres amérindiennes, Société d'ethnologie, Nanterre, pp. 431-479. 
Villa Rojas Alfonso

1978 Los elegidos de Dios, Instituto Nacional Indigenista, Mexico [1945, The Maya of East Central Quintana Roo, Carnegie Institution, Washington, coll. « Publication » 559].

Vogt Evon Z.

1966

"Ancestor worship in Zinacantan religion », in Actas y Memorias del XXXVI Congreso Internacional de Americanistas, Séville, vol. 3, pp. 281285.

1969 Zinacantan, a Maya Community in the Highlands of Chiapas, The Belknap Press of Harvard University Press, Cambridge, Mass.

Wolf Arthur P., ed.

1974

Religion and Ritual in Chinese Society, Stanford University Press, Stanford. 